\title{
Détermination de l'altitude du Zéro des stations hydrométriques en Amazonie brésilienne. Application aux lignes d'eau des Rios Negro, Solimões et Amazone Zero altitude determination for hydrometric stations in the Brazilian Amazon. Application to the water surfaces of Rios Negro, Solimões and Amazon
}

\author{
Jacques Callède, Daniel Medeiros Moreira et Stéphane Calmant
}

Volume 26, numéro 2, 2013

Reçu le 11 novembre 2012, accepté le 20 mars 2013

URI : https://id.erudit.org/iderudit/1016065ar

DOI : https://doi.org/10.7202/1016065ar

Aller au sommaire du numéro

Éditeur(s)

Université du Québec - INRS-Eau, Terre et Environnement (INRS-ETE)

ISSN

1718-8598 (numérique)

Découvrir la revue

Citer cet article

Callède, J., Moreira, D. M. \& Calmant, S. (2013). Détermination de l'altitude du Zéro des stations hydrométriques en Amazonie brésilienne. Application aux lignes d'eau des Rios Negro, Solimões et Amazone. Revue des sciences de l'eau / Journal of Water Science, 26(2), 153-171. https://doi.org/10.7202/1016065ar

\section{Résumé de l'article}

Le rattachement altimétrique d'une station hydrométrique à un repère du Nivellement Général est habituellement effectué dans le but de connaître, lorsqu'il y a plusieurs stations, la pente de la ligne d'eau. Jusqu'à la fin de la décennie 2000, les stations hydrométriques amazoniennes n'étaient pas rattachées car le manque de réseau routier était un obstacle majeur au classique nivellement direct. Mais la fin de cette décennie a vu l'expansion de l'altimétrie satellitaire, et plus particulièrement celle de la méthode utilisant le GPS associé à l'utilisation du modèle de géoïde EGM08. Cette nouvelle technique est particulièrement précieuse pour niveler les stations hydrométriques du bassin amazonien. Nous avons traité ici les stations hydrométriques de l'Amazone depuis son embouchure jusqu'à la frontière Brésil/Pérou et celles des rios Negro et Solimões pour leurs parties brésiliennes. Les pentes moyennes de ces différents biefs décroissent normalement sur le Rio Solimões et l'Amazone, tandis que le bief Moura-Manaus, sur le Rio Negro, présente anormalement une pente très faible $\left(6 \mathrm{~mm} \cdot \mathrm{km}^{-1}\right)$ au risque de provoquer des inversions d'écoulement. Le Bas Amazone est raccordé à l'altitude donnée par la trace de l'orbite 202 des satellites Topex-Poseidon et Jason-1 mais malheureusement nous ne disposons que d'une seule année de relevés limnigraphiques sur ce tronçon. Les comparaisons des pentes de la ligne d'eau avec d'autres fleuves (Volga, Danube, Nil, Mississipi et Rhin) indiquent que la pente de l'Amazone est, avec en moyenne $21 \mathrm{~mm} \cdot \mathrm{km}^{-1} \mathrm{de}$ Tabatinga (frontière Brésil/Pérou) à l'Océan Atlantique (soit sur $3000 \mathrm{~km}$ ) deux fois moindre que celle de la Volga (qui présente la ligne d'eau de pente la plus faible sur les cinq fleuves étudiés). Ceci, associé au fait que le fond du lit de l'Amazone est, sur $2000 \mathrm{~km}$ à partir de son embouchure, à une quarantaine de mètres en-dessous du niveau de l'océan, nous conduirait à penser que l'écoulement du fleuve subit une poussée des eaux amont, issues de la Cordillère des Andes. 


\title{
DÉTERMINATION DE L'ALTITUDE DU ZÉRO DES STATIONS HYDROMÉTRIQUES EN AMAZONIE BRÉSILIENNE. APPLICATION AUX LIGNES D'EAU DES RIOS NEGRO, SOLIMÓES ET AMAZONE
}

Zero altitude determination for hydrometric stations in the Brazilian Amazon.

Application to the water surfaces of Rios Negro, Solimões and Amazon

\section{JACQUES CALLÈDE*, DANIEL MEDEIROS MOREIRA ${ }^{1}$, STÉPHANE CALMANT²}

\author{
*Retraité IRD, QE15, conj. S, casa 51, Guará II, 71050-191 Brasília (Distrito Federal), Brasil, cld@apis.com.br. \\ ${ }^{1}$ CPRM, avenida Pasteur, 404, Urca, CEP : 22290-240 Rio de Janeiro (RJ), Brasil \\ ${ }^{2}$ Laboratoire d'études en géophysique et océanographie spatiales (LEGOS) \\ 18, av. Edouard Belin, 31401 Toulouse cedex 9, France
}

Reçu le 11 novembre 2012, accepté le 20 mars 2013

\section{RÉSUMÉ}

Le rattachement altimétrique d'une station hydrométrique à un repère du Nivellement Général est habituellement effectué dans le but de connaitre, lorsqu'il y a plusieurs stations, la pente de la ligne d'eau. Jusqu'à la fin de la décennie 2000, les stations hydrométriques amazoniennes n'étaient pas rattachées car le manque de réseau routier était un obstacle majeur au classique nivellement direct. Mais la fin de cette décennie a vu l'expansion de l'altimétrie satellitaire, et plus particulièrement celle de la méthode utilisant le GPS associé à l'utilisation du modèle de géoïde EGM08. Cette nouvelle technique est particulièrement précieuse pour niveler les stations hydrométriques du bassin amazonien. Nous avons traité ici les stations hydrométriques de l'Amazone depuis son embouchure jusqu'à la frontière Brésil/Pérou et celles des rios Negro et Solimóes pour leurs parties brésiliennes. Les pentes moyennes de ces différents biefs décroissent normalement sur le Rio Solimóes et l'Amazone, tandis que le bief Moura-Manaus, sur le Rio Negro, présente anormalement une pente très faible $\left(6 \mathrm{~mm} \bullet \mathrm{km}^{-1}\right)$ au risque de provoquer des inversions d'écoulement. Le Bas Amazone est raccordé à l'altitude donnée par la trace de l'orbite 202 des satellites Topex-Poseidon et Jason-1 mais malheureusement nous ne disposons que d'une seule année de relevés limnigraphiques sur ce tronçon. Les comparaisons des pentes de la ligne d'eau avec d'autres fleuves (Volga, Danube, Nil, Mississipi et Rhin) indiquent que la pente de l'Amazone est, avec en moyenne $21 \mathrm{~mm} \bullet \mathrm{km}^{-1}$ de Tabatinga (frontière Brésil/Pérou) à l'Océan Atlantique (soit sur $3000 \mathrm{~km}$ ) deux fois moindre que celle de la Volga (qui présente la ligne d'eau de pente la plus faible sur les cinq fleuves étudiés). Ceci, associé au fait que le fond du lit de l'Amazone est, sur $2000 \mathrm{~km}$ à partir de son embouchure, à une quarantaine de mètres en-dessous du niveau de l'océan, nous conduirait à penser que l'écoulement du fleuve subit une poussée des eaux amont, issues de la Cordillère des Andes.

Mots-clés : nivellement, altimétrie satellitaire, GPS, bassin amazonien, Bas Amazone, Rio Negro, Rio Solimóes, écoulement. 


\section{ABSTRACT}

The altimetric relation of a hydrometric station to the General Levelling mark is usually performed in order to know, when there are several stations, the slope of the water line. Until the end of the 2000s, Amazonian hydrometric stations were not related because the lack of roads was a major obstacle to classical direct levelling. However, the end of this decade has seen the expansion of satellite altimetry and more particularly the development of the method using GPS (Global Positioning System) associated with the use of the geoid model EGM08. This new technique is particularly valuable for level gauging stations in the Amazon Basin. Here we have treated hydrometric stations of the Amazon from its mouth to the Brazil/Peru border and those of the rivers Negro and Solimóes in their Brazilian part. Average slopes of these reaches decrease normally for the Rios Solimôes and Amazon, whereas for the Moura/Manaus reach on the Rio Negro, the abnormally low slope $\left(6 \mathrm{~mm} \bullet \mathrm{km}^{-1}\right)$ may cause inverted flow. The lower Amazon is connected to the altitude given by the trace of orbit 202 of the Topex-Poseidon and Jason-1 satellites, but unfortunately we only have one year of gauging readings for this reach. Comparisons of the water slopes with other rivers (Volga, Danube, Nile, Mississippi and Rhine) indicate that the slope of the Amazon is, with an average of $21 \mathrm{~mm} \bullet \mathrm{km}^{-1}$ from Tabatinga (Brazil/Peru border) to the Atlantic Ocean (a distance of $3000 \mathrm{~km}$ ), two times less than that of the Volga (which has the lowest water line slope among the five rivers studied). This, coupled with the fact that the bed of the Amazon is, for about $2000 \mathrm{~km}$ from its mouth, about forty meters below the level of the ocean, would lead us to believe that the river's flow is propelled by water arriving from upstream, from the Andes.

Keywords : levelling, satellite altimetry, GPS, Amazon Basin, Lower Amazon, Rio Negro, Rio Solimóes, stream flow.

\section{INTRODUCTION}

Quand il en a la possibilité, l'hydrologue rattache toujours sa station hydrométrique à un repère du Nivellement Général. Dans les Monographies hydrologiques de l'IRD (Institut de Recherche pour le développement), anciennement ORSTOM (Office de la Recherche Scientifique et Technique OutreMer), ce rattachement figure habituellement dans le chapitre "Équipement hydrométrique " (CALLÈDE et al., 2010). Sur les grands cours d'eau, cela permet de déterminer la pente de la ligne d'eau entre deux stations, qui est un paramètre important en hydraulique fluviale et en géomorphologie. Cette précaution est devenue si pressante que, par exemple, en France et durant le $\mathrm{XX}^{\mathrm{e}}$ siècle, le profil en long de 1356 cours d'eau a été spécialement établi (avec pose de repères de nivellement) soit par le Service des Ponts et Chaussées, soit par celui du Nivellement Général de la France ou encore par celui des Forces Hydrauliques, avec une priorité pour les rivières aménageables en vue de la production hydroélectrique (CROZIER, 1938; DE MARGERIE, 1910).

L'étude des variations de la pente de la ligne d'eau sert aux géomorphologues, par exemple, pour différencier les zones d'érosion de celles de dépôt des sédiments.

Tout cela implique l'existence d'un réseau de nivellement de précision, réalisé au niveau à lunette le long des routes ou des voies ferrées.

Au Brésil, ce travail long et fastidieux est réalisé par les équipes spécialisées dépendantes de la Direction des Géosciences de l'IBGE (Instituto Brasileiro de Geografia e Estatistica - Institut Brésilien de Géographie et de Statistique) (IBGE, 2012). Malheureusement, les routes sont bien rares en Amazonie, où la voie de communication primordiale reste toujours la rivière. La figure 1 représente les mailles de nivellement de précision du Brésil. Le contraste entre l'Amazonie (fond jaune clair, à gauche du méridien $-050^{\circ} \mathrm{W}$ et au-dessus du parallèle $-15^{\circ} \mathrm{S}$ ) et le reste du pays saute aux yeux : en Amazonie, il n'y a que très peu de mailles, mis à part l'axe Porto Velho-Manaus-Boa Vista, la route Transamazonienne sud et l'axe Cuiabá-Porto Velho. Sauf pour les sites de Manaus, Manacapuru, Itacoataria, Santarém, Altamira et Porto Velho, situés le long des mailles de nivellement où un rattachement au nivellement reste possible, aucune liaison des stations hydrométriques au réseau brésilien n'a été faite : les échelles limnimétriques sont simplement nivelées par rapport à des repères (généralement des bornes) de façon à pouvoir contrôler, comme le recommandait ROCHE (1963), la bonne stabilité des éléments.

Jusqu'à la dernière décennie du $\mathrm{XX}^{\mathrm{e}}$ siècle, seul le nivellement barométrique pouvait donner la valeur du zéro d'une station hydrométrique, au mieux au mètre près, et encore, en altitude relative. Ceci impliquait d'utiliser deux altimètres du type Thommen, Paulin ou Wallace et Tiernan, l'un restant à poste fixe (pour suivre la marée barométrique) tandis que l'autre se déplaçait sur le terrain (ERWES, 1975).

Les techniques du nivellement spatial apparaissent durant cette dernière décennie du XX ${ }^{\mathrm{e}}$ siècle. Elles deviennent vraiment opérationnelles en 2008 et permettent d'obtenir des altitudes absolues avec une précision quasi décimétrique. Les valeurs que nous présentons ici sont parmi les premières déterminations par altimétrie satellitaire des altitudes des zéros de quelques stations hydrométriques principales du bassin amazonien.

La qualité première que tout hydrologue recherche dans une station hydrométrique est sa stabilité verticale. Peu lui importe que, suivant les différents systèmes de nivellement, l'altitude de 


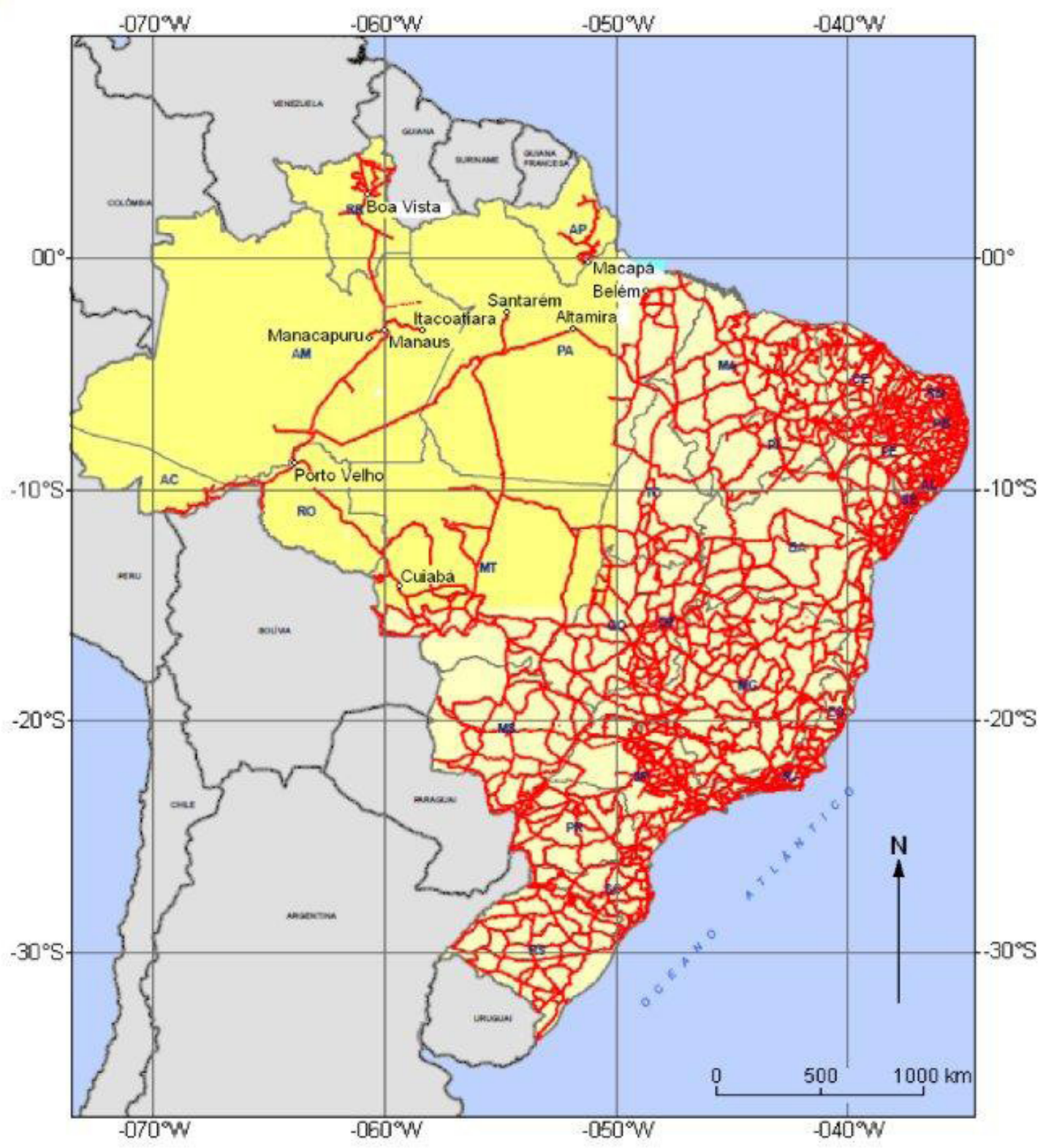

Figure 1. Les mailles de nivellement de précision au Brésil (document IBGE). The mesh of precision levelling in Brazil (IBGE document).

son zéro varie : le principal est que l'échelle hydrométrique n'ait pas varié en hauteur. Un bel exemple de station hydrométrique stable est donné par celle de Manaus, installée à son Port en 1902 (historique réalisé d'après un document, probablement écrit par un hydrographe de la DHN (Diretoria de Hidrografia e Navigação - Direction de l'Hydrographie et de la Navigation) en date du 18 décembre 1980.

En 1902, cette station consistait en une règle graduée dont la base était cotée $+62,61 \mathrm{~m}$ et le sommet $+92,28 \mathrm{~m}$, valeurs établies suite à un nivellement barométrique du sommet.

En 1998, cette station est toujours identique à celle de 1902, mis à part que, depuis septembre 1988, l'observateur retranche systématiquement $62,61 \mathrm{~m}$ à la lecture, ce qui revient à considérer la station comme étant graduée fictivement de 0 à 29,67 m, le zéro (fictif) de la station étant ainsi à l'altitude + 62,61 m. Les archives ont été modifiées en conséquence.

Cependant, depuis 1902, la valeur de l'altitude de son zéro a varié au cours du temps :

- en 1915, un second nivellement barométrique était réalisé. L'altitude du sommet de l'échelle est déterminée à $+35,873 \mathrm{~m}$, ce qui donne le zéro fictif de l'échelle à l'altitude $+6,20 \mathrm{~m}$.

Différents nivellements de contrôle du rattachement sont réalisés tant par la DHN que par la CPRM (Companhia de 
Pesquisa de Recursos Minerais - Compagnie de Recherche de Ressources Minérales, chargée da la gestion du réseau hydrologique) avec des fermetures allant de 1 à $24 \mathrm{~mm}$;

- en 1998, suite aux visites que nous avons faites à la Direction de la Géodésie, dépendante de la Direction des Géosciences de l'IBGE (Instituto Brasileiro de Geografia e Estatistica - Institut Brésilien de Géographie et de Statistique) à Rio de Janeiro, nous avons déterminé, dans le système de nivellement de précision de l'IBGE, l'altitude du zéro (fictif) de la station de Manaus à - 4,17 m puis, le 15 avril 1999, à - 3,70 m suite à une modification due à la compensation du cheminement;

- KOSUTH (2006) indique un zéro (fictif) à l'altitude - 6,90 m en utilisant les mesures GPS (Global Positioning System - Système mondial de Positionnement) et le modèle de Géoïde EGM96 (Earth Gravitational Model 1996 - Modèle de Gravité 1996) et - 7,51 m en utilisant le satellite TOPEX (TOPography EXPerimental - Expérimental pour la Topographie) - Poseidon (nom du radar altimétrique embarqué) et l'EGM96;

- Finalement, notre détermination du zéro de la station faite en 2008 (MEDEIROS MOREIRA, 2010), utilisant les mesures GPS et l'EGM08 ((Earth Gravitational Model 2008 - Modèle de Gravité 2008), donne un zéro (fictif) à l'altitude de - 6,92 m.

Ceci montre le peu d'exactitude du nivellement barométrique en valeur absolue et les difficultés rencontrées lors du nivellement de " précision " de l'IBGE, notamment dans le franchissement des grands cours d'eau dépourvus de pont.

Dans cette étude nous examinerons, après un bref aperçu sur l'altimétrie satellitaire, la méthode de détermination de l'altitude du zéro de la station hydrométrique et son application aux pentes de la ligne d'eau des Rios Negro, Solimôes (dans leur partie brésilienne) et Amazone.

Ces travaux s'intègrent dans le cadre des recherches du Laboratoire ORE-HYBAM (Contrôles géodynamique, hydrologique et biogéochimique de l'érosion/altération et des transferts de matière dans le bassin de l'Amazone) relevant du GET (Géosciences, Environnement, Toulouse).

\subsection{Brefaperçu sur l'altimétrie satellitaire}

La dernière décennie du $\mathrm{XX}^{\mathrm{e}}$ siècle est marquée par l'apparition de satellites spécialisés dans la détermination de l'altitude de la surface des océans avec le satellite TOPEX-Poseidon, mis sur orbite en 1992, suivi par Jason-1 et Jason-2, ERS (European Remote Sensing - Satellite Européen d'Observation de la Terre) 1 et 2, puis enfin ENVISAT (ENVIronment SATellite - Satellite pour l'Environnement). Ces satellites sont utilisés en Amazonie pour déterminer les altitudes de l'eau à des stations "virtuelles " situées sous la trace orbitale (CALMANT et SEYLER, 2006; KOSUTH et al., 2006 et 2007; LEON, 2006; SANTOS DA SILVA et al., 2010; SEYLER et al., 2007; ZHANG et al., 2010). Cette technique, indépendante et complémentaire, ne sera pas développée ici.

Le système satellitaire GPS apparaît un peu plus tôt (1978). Développé à cette époque pour les besoins de l'US Army, il devient libre d'accès en 1994 et sa précision planimétrique lui ouvre l'accès des applications géodésiques, puis du nivellement de précision (DUQUENNE, 2010; RESSOURCES NATURELLES CANADA, 2012). C'est ce système qui est utilisé dans notre étude.

\subsection{Détermination de l'altitude du zéro des stations hydrométriques}

Á l'origine destiné à la localisation planimétrique, le GPS s'avère capable d'assumer la détermination de la hauteur ellipsoïdale d'un point, c'est-à-dire sa hauteur par rapport à l'ellipsoïde de référence, ici le WGS84 (Word Geodetic System - Système Géodésique Mondial, révision de 1984) (distance ME, Figure 2). Sa précision quasi centimétrique (CRÉTAUX et al., 2011; DE CASTRO, 2002; DUQUENNE, 2010; RESSOURCES NATURELLES CANADA, 2012) l'a rendu indispensable pour les opérations de maintenance ou de compensation des réseaux de nivellement généraux (DUQUENNE， 2010; RESSOURCES NATURELLES CANADA, 2012).

Ceci implique certaines astreintes : il faut stationner le point dont on veut déterminer l'altitude avec un récepteur GPS géodésique bi-fréquences et enregistrer les mesures durant plusieurs heures, puis effectuer les quelques corrections liées aux conditions de propagations troposphérique et ionosphérique (DE CASTRO, 2002; KOUBA, 2009; LEICK, 2003; MEDEIROS MOREIRA, 2010; MONICO, 2000).

Par contre, ce qui nous intéresse c'est l'altitude de ce point, c'est-à-dire la hauteur prise par rapport au géoöde (distance MG, Figure 2) qui est la surface moyenne des océans. Or, jusqu'en 2008, le passage de l'ellipsoïde au géoïde (distance EG, Figure 2) se faisait avec un modèle EGM96 dont la précision n'était que de $\pm 1,65 \mathrm{~m}$ (KOSUTH, 2007), ceci à cause du manque de mesures gravimétriques terrestres dans certaines régions du Globe (dont l'Amazonie). C'est la raison pour laquelle le nivellement satellitaire, en valeurs absolues, n'est utilisé qu'à partir de 2008. Nous avons cependant reporté, dans le tableau 1, les altitudes des zéros que KOSUTH et al. (2006) ont déterminées avec le modèle EGM96 pour l'Amazone et le Rio Solimões, tant avec le GPS qu'avec Topex-Poseidon.

Il faut attendre les résultats des mesures de la gravité effectuées par les satellites jumeaux GRACE (Gravity Recovery 


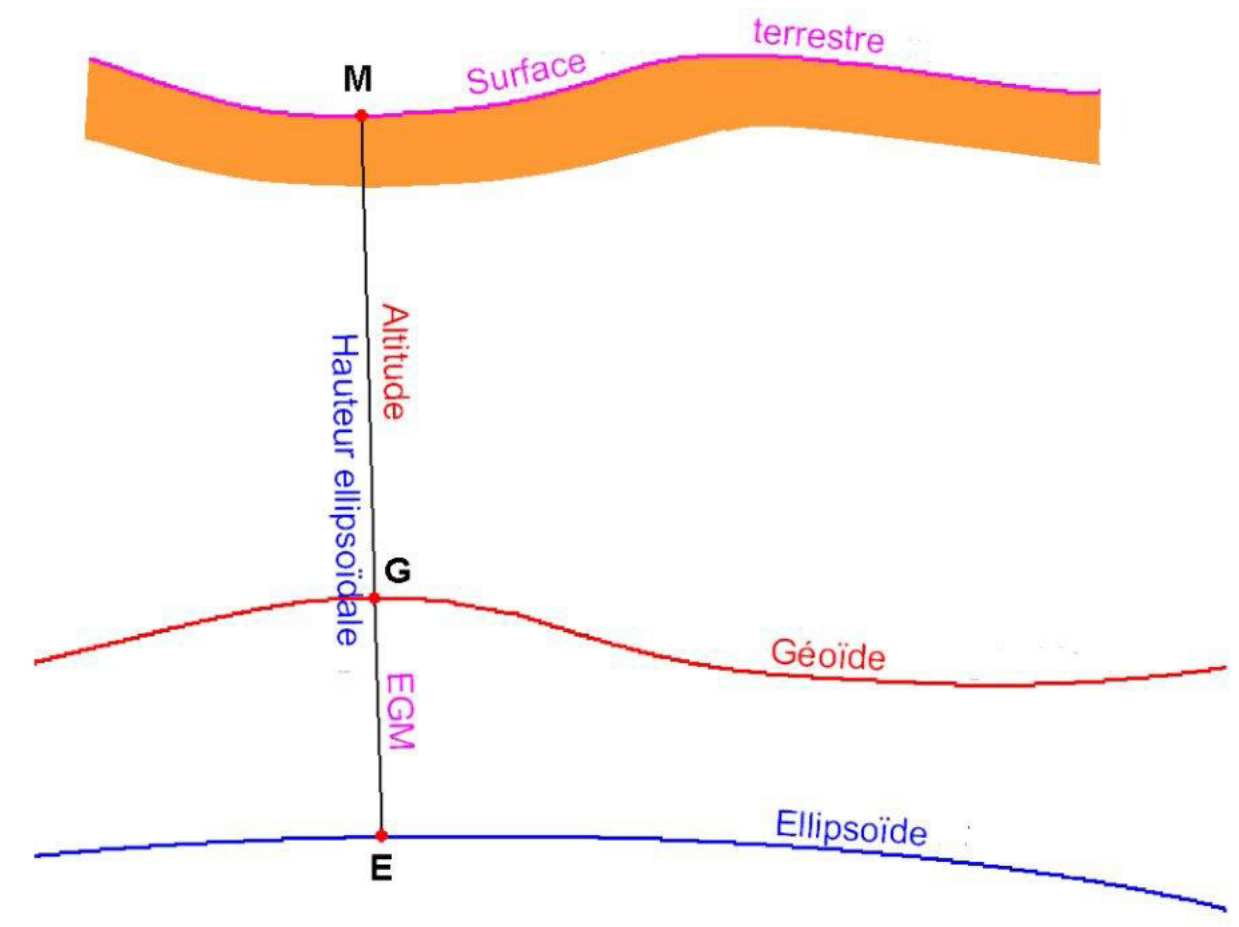

Figure 2. Ellipsoïde, géoïde et surface terrestre. Ellipsoid, geoid and Earth's surface.

And Climate Experiment - Récupération de la gravité et expérimentation climatique), mis en orbite en mars 2002, pour réaliser, en 2008, le modèle de gravité EGM08 qui calcule l'éloignement géoïde/ellipsoïde avec un écart-type de $\pm 13 \mathrm{~cm}$ (ARABELOS et TSCHERNING, 2010; BLITZKOW et DE MATOS, 2009; PAVLIS et al., 2008). Nous utilisons ici la version de ce modèle qui donne la hauteur, en un point, de l'ondulation du géoïde par rapport à l'ellipsoïde WGS84 (EGM2008-WGS84 Version), disponible à la National Geospatial Intelligence Agency (USA) sur le site : http://earthinfo.nga.mil/GandG/wgs84/gravitymod/egm2008/egm08_ wgs84.html.

\section{MATÉRIEL ET MÉTHODES}

\subsection{Détermination du zéro des stations hydrométriques}

Les altitudes des stations hydrométriques principales des Rios Negro, Solimóes et Amazone en amont de Santarém, ont été déterminées par CALMANT et MEDEIROS MOREIRA lors de campagnes de mesures effectuées en 2008 et 2009.

Pour la partie située en aval de Santarém, nous utilisons également les hauteurs ellipsoïdales, obtenues en 1998 et
1999 par l'IBGE pour le compte de KOSUTH, et le modèle EMG2008-WGS84 Version pour calculer l'ondulation du géoïde.

\subsubsection{Résultats}

La figure 3 montre la position des stations hydrométriques nivelées par GPS tandis que le tableau 1 récapitule les altitudes des zéros des stations hydrométriques brésiliennes.

Ces stations sont classées par bassin (Rio Negro, Rio Solimóes, Amazone et Bas Amazone) et, pour chacun, de l'amont vers l'aval.

Pour l'Amazone et le Rio Solimões, le tableau 1 présente également les valeurs tirées de l'étude de KOSUTH et al. (2006), afin de montrer les écarts entre les modèles de géoïde EGM96 et EGM08 et entre les résultats GPS et Topex-Poseidon. Le maigre échantillon ne porte que sur 10(11) valeurs, les différences sur le Bas Amazone étant trop importantes, n'ont pas été prises en compte. En prenant pour base nos altitudes obtenues avec le modèle EGM08, les écarts entre les altitudes GPS varient entre $+1,23 \mathrm{~m}$ (Téfé) et $-0,05 \mathrm{~m}$ (Itacoatiara) avec une valeur moyenne de $+0,59 \mathrm{~m}$ et un écart-type de $\pm 44 \mathrm{~cm}$. Pour les altitudes Topex-Poséidon, les différences varient entre $+1,11 \mathrm{~m}$ (Santarém) et - 0,16 m (Itacoatiara) avec une valeur moyenne de $+0,49 \mathrm{~m}$ et un écart-type de $\pm 36 \mathrm{~cm}$. 
Tableau 1. Altitude du zéro des stations hydrométriques de l'Amazonie brésilienne, nivelées par GPS.

Table 1 Zero elevation of the hydrometric stations in the Brazilian Amazon, leveled by GPS.

\begin{tabular}{|c|c|c|c|c|c|}
\hline \multirow{3}{*}{ Station } & \multirow{3}{*}{ Latitude } & \multicolumn{4}{|c|}{ Altitude (m) } \\
\hline & & \multirow[t]{2}{*}{ Longitude } & \multirow{2}{*}{$\begin{array}{l}\text { Calmant } \\
\text { EGM08 }\end{array}$} & \multicolumn{2}{|c|}{ Kosuth } \\
\hline & & & & GPS & $\mathrm{T} / \mathrm{P}$ \\
\hline Rio Negro & (source : S. CALMANT) & & & & \\
\hline São Gabriel da Cachoeira & $00^{\circ} 08^{\prime} 10,0^{\prime \prime} \mathrm{N}$ & $067^{\circ} 05^{\prime} 04,9^{\prime \prime} \mathrm{W}$ & 43,42 & & \\
\hline Tapurucuara & $00^{\circ} 25^{\prime} 13,1^{\prime \prime} \mathrm{S}$ & $065^{\circ} 00^{\prime} 55,1^{\prime \prime} \mathrm{W}$ & 25,33 & & \\
\hline Barcelos & $00^{\circ} 57^{\prime} 56,9^{\prime \prime} \mathrm{S}$ & $062^{\circ} 55^{\prime} 52,0^{\prime \prime} \mathrm{W}$ & 15,33 & & \\
\hline Moura & $01^{\circ} 27^{\prime} 24,1^{\prime \prime} \mathrm{S}$ & $061^{\circ} 38^{\prime} 04,9^{\prime \prime} \mathrm{W}$ & 9,07 & & \\
\hline Manaus & $03^{\circ} 08^{\prime} 12,1^{\prime \prime} \mathrm{S}$ & $060^{\circ} 01^{\prime} 36,8^{\prime \prime} \mathrm{W}$ & $-6,92$ & $-6,90$ & $-7,51$ \\
\hline Rio Solimōes & (source : S. CALMANT) & & & & \\
\hline Tabatinga & $04^{\circ} 14^{\prime} 04,9^{\prime \prime} \mathrm{S}$ & $069^{\circ} 56^{\prime} 40,9^{\prime \prime} \mathrm{W}$ & 56,45 & 55,57 & 56,07 \\
\hline São Paulo de Olivença & $03^{\circ} 27^{\prime} 00,0^{\prime \prime} \mathrm{S}$ & $068^{\circ} 45^{\prime} 00,0^{\prime \prime} \mathrm{W}$ & 45,52 & 44,47 & 44,92 \\
\hline Fonte Boa & $02^{\circ} 29^{\prime} 29,0^{\prime \prime} \mathrm{S}$ & $066^{\circ} 03^{\prime} 42,1^{\prime \prime} \mathrm{W}$ & 22,85 & 21,96 & 22,3 \\
\hline Téfé & $03^{\circ} 22^{\prime} 32,9^{\prime \prime} \mathrm{S}$ & $064^{\circ} 39^{\prime} 16,9^{\prime \prime} \mathrm{W}$ & 22,16 & 20,93 & 21,81 \\
\hline Itapeua & $04^{\circ} 03^{\prime} 28,1^{\prime \prime} \mathrm{S}$ & $063^{\circ} 01^{\prime} 40,1^{\prime \prime} \mathrm{W}$ & 14,40 & 13,74 & 13,97 \\
\hline Manacapuru & $03^{\circ} 18^{\prime} 38,2^{\prime \prime} \mathrm{S}$ & $060^{\circ} 36^{\prime} 33,8^{\prime \prime} \mathrm{W}$ & 4,37 & & 3,96 \\
\hline Manaus & $03^{\circ} 08^{\prime} 12,1^{\prime \prime} \mathrm{S}$ & $060^{\circ} 01^{\prime} 36,8^{\prime \prime} \mathrm{W}$ & $-6,92$ & $-6,90$ & $-7,51$ \\
\hline Amazone & (source : S. CALMANT) & & & & \\
\hline Manaus & $03^{\circ} 08^{\prime} 12,1^{\prime \prime} \mathrm{S}$ & $060^{\circ} 01^{\prime} 36,8^{\prime \prime} \mathrm{W}$ & $-6,92$ & $-6,90$ & $-7,51$ \\
\hline Jatuarana & $03^{\circ} 03^{\prime} 47,9^{\prime \prime} \mathrm{S}$ & $059^{\circ} 38^{\prime} 52,1^{\prime \prime} \mathrm{W}$ & 3,11 & & \\
\hline Itacoatiara & $03^{\circ} 08^{\prime} 04,9^{\prime \prime} \mathrm{S}$ & $058^{\circ} 28^{\prime} 59,9^{\prime \prime} \mathrm{W}$ & $-3,70$ & $-3,65$ & $-3,54$ \\
\hline Parintins & $02^{\circ} 38^{\prime} 01^{\prime \prime} \mathrm{S}$ & $056^{\circ} 45^{\prime} 07^{\prime \prime} \mathrm{W}$ & 5,42 & 4,92 & 4,74 \\
\hline Óbidos & $01^{\circ} 55^{\prime} 09,1^{\prime \prime} \mathrm{S}$ & $055^{\circ} 30^{\prime} 47,2^{\prime \prime} \mathrm{W}$ & 3,58 & 2,97 & 2,56 \\
\hline Santarém & $02^{\circ} 24^{\prime} 50,2^{\prime \prime} \mathrm{S}$ & $054^{\circ} 44^{\prime} 16,0^{\prime \prime} \mathrm{W}$ & 2,95 & 2,77 & 1,84 \\
\hline Bas Amazone & (source : IBGE, J. CALI & & & & \\
\hline Santarém & $02^{\circ} 24^{\prime} 50,2^{\prime \prime} \mathrm{S}$ & $054^{\circ} 44^{\prime} 16,0^{\prime \prime} \mathrm{W}$ & 2,95 & 2,77 & 1,84 \\
\hline Prainha & $01^{\circ} 48^{\prime} 31^{\prime \prime} \mathrm{S}$ & $053^{\circ} 28^{\prime} 52^{\prime \prime} \mathrm{W}$ & 1,14 & 6,29 & 0,34 \\
\hline Almeirim & $01^{\circ} 31^{\prime} 57^{\prime \prime} \mathrm{S}$ & $052^{\circ} 24^{\prime} 39^{\prime \prime} \mathrm{W}$ & $-0,61$ & 3,77 & 0,19 \\
\hline Gurupá & $01^{\circ} 24^{\prime} 18^{\prime \prime} \mathrm{S}$ & $051^{\circ} 38^{\prime} 48^{\prime \prime} \mathrm{W}$ & 0,51 & 2,80 & $-0,29$ \\
\hline Porto Santana & $00^{\circ} 03^{\prime} 39^{\prime \prime} \mathrm{S}$ & $051^{\circ} 09^{\prime} 36^{\prime \prime} \mathrm{W}$ & $-2,89$ & 4,39 & \\
\hline
\end{tabular}

(Les altitudes "Kosuth" sont déterminées par rapport au géoïde calculé par le modèle EGM06)

Ces dispersions sont nettement plus faibles que la précision annoncée par KOSUTH $( \pm 1,65 \mathrm{~m})$.

\subsubsection{Cas des stations de l'Amazone}

Le zéro des stations de Parintins, Óbidos et Santarém a été déterminé deux fois : par l'IBGE en 1999 et par CALMANT vers 2008. Les écarts entre les deux déterminations sont, respectivement, de 13, 16 et $8 \mathrm{~cm}$. Pour Parintins et Óbidos, nous avons adopté la mesure effectuée par CALMANT (qui placel'antenne du GPS directement sur l'échelle limnimétrique) et pour Santarém, nous avons choisi de prendre la valeur moyenne des deux déterminations.

\subsection{Méthode de calcul des pentes}

Le Bas Amazone mis à part (voir $\$ 2.3$ ), le calcul des pentes, sous Excel, s'effectue en année hydrologique (01 novembre à
31 octobre), au pas de temps journalier et par biefs consécutifs (un bief étant la portion de cours d'eau comprise entre deux stations hydrométriques successives).

Après avoir rassemblé les données (soit du fichier hydrométrique de l'ORE-HYBAM, disponible à http://www. ore-hybam.org/, soit celui de l'ANA (Agência Nacional da Águas - Agence Nationale de l'Eau) disponibles à http://www. ana.gov.br/portalsnirh/), la continuité de la séquence est vérifiée et les hauteurs manquantes sont complétées par interpolation ou par reconstitution avec les stations aval et amont (cas de Moura en 1991). Les altitudes journalières de l'eau aux stations amont et aval sont ensuite calculées en utilisant les déterminations du zéro des échelles de CALMANT (Tableau 1), ainsi que la différence de niveau amont-aval. Quand cette dénivelée est négative, les relevés de hauteur d'eau sont soigneusement vérifiés avec les documents originaux quand ils existent et avec les commentaires des hydrologues de la CPRM lors de leurs 


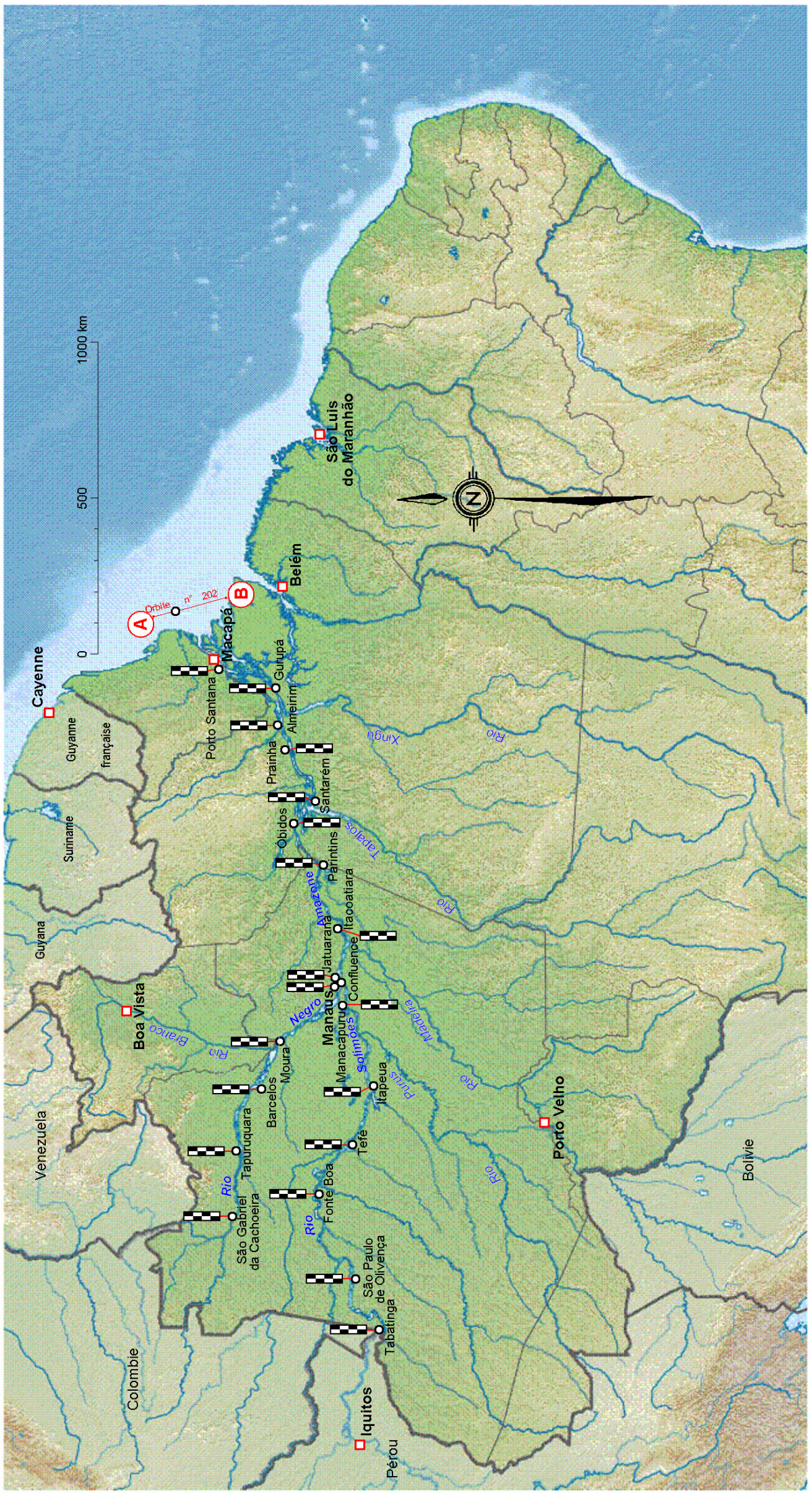

Figure 3. Emplacement des stations hydrométriques de l'Amazonie brésilienne nivelées par GPS.

Location of the Brazilian Amazon hydrometric stations leveled by GPS. 
tournées d'inspection (http://visualizador.ana.gov.br:8080/ VisualizadorWebLogic/ ou http://www2.ana.gov.br puis HydroWeb) et ceux de la Télétransmission (http://www2.ana. gov.br/portalsnirh).

Le tableur Excel permet ensuite de déterminer, année par année :

- la dénivelée moyenne;

- la dénivelée maximale;

- la dénivelée minimale.

La longueur du bief est déterminée avec les cartes au 1/1 000000 de l'IBGE, en suivant les sinuosités du cours d'eau (méthode de la bande de papier et du piquoir). Après récapitulation et moyennes, les pentes (en $\mathrm{mm} \bullet \mathrm{km}^{-1}$ ) sont calculées. Une homogénéisation sur la période 1980/2009 (30 années) est effectuée.

\subsection{La ligne d'eau du Bas Amazone}

L'influence de la marée océanique se fait sentir jusqu'à Óbidos où, en basses eaux, le marnage lié à la marée est de $10 \mathrm{~cm}$ (CALLÈDE et al., 2001).

Santarém est la dernière station la plus en aval prise en compte par l'ANA. Toutes les autres stations, entre Santarém et Porto Santana (Macapá) sont (ou ont été) lues par la DHN et ne servent que pour les besoins de la navigation fluviale.

Pour une étude sur l'influence de la marée sur le Bas Amazone, KOSUTH avait installé et exploité, de 1999 à 2001, une série de limnigraphes à pression OTT "Orphimedes » (KOSUTH et al., 2009) avec une mesure toutes les 30 minutes. Seule l'année 2000 est à peu près complète.

Pour supprimer la composante " marée ", nous avons commencé par déterminer la hauteur moyenne journalière en calculant le quotient des 48 mesures journalières. Nous constatons qu'il persiste encore, à toutes les stations hydrométriques du Bas Amazone, une oscillation résiduelle, parfaitement synchrone avec les phases de la Lune (Figure 4) et dont l'amplitude décroissait de l'aval vers l'amont. Cette anomalie est lissée avec soit une moyenne mobile centrée de degré 28 , soit une courbe de tendance polynomiale d'ordre 4, les résultats étant quasi identiques.

\subsubsection{Raccordement à l'Océan Atlantique}

Grâce à FROSSARD, le raccordement à l'Océan Atlantique est fait en utilisant les altitudes de l'océan de l'orbite 202 de TOPEX-Poseidon et Jason-1, débarrassées de l'influence de la marée océanique.

Ces altitudes sont données par rapport à l'ellipsoïde du Centre National d'Études Spatiales / Archivage, Validation, Interprétation, des données de Satellites Océanographiques (CNES/AVISO) qui diffère légèrement de WGS84 (BERCHER, 2008).

Ellipsoide CNES/AVISO

Demi-Grand axe majeur : $6378136,3 \mathrm{~m}$ et aplatissement : 1/298,257, d'où un demi-grand axe mineur de 6356751,6 m;

WGS84

Demi-Grand axe majeur : $6378137,0 \mathrm{~m}$ et aplatissement : $1 / 298,257223563$, d'où un demi-grand axe mineur de $6356752,31 \mathrm{~m}$,

ce qui veut dire que la différence entre l'ellipsoïde WGS84 et celui du CNES/AVISO est de $+0,7 \mathrm{~m}$ à l'Équateur et + 0,71 $\mathrm{m}$ aux Pôles et, par conséquent, nous pouvons la considérer comme constante. Plus simplement, nous dirons que les hauteurs par rapport à l'ellipsoïde données par les satellites TOPEX-Poséidon + Jason-1 doivent être diminuées de $70 \mathrm{~cm}$ pour les ramener dans le Système WGS84. En plus, ces altitudes sont à diminuer de $3 \mathrm{~cm}$ pour tenir compte des anomalies de surface de la mer en 2000 (d'après un second fichier " anomalies de surface de la mer ", fourni également par F. FROSSARD).

La figure 5 représente le profil en long de l'Océan Atlantique suivant la trace au sol de l'orbite $\mathrm{n}^{\circ} 202$ de TOPEX-Poseidon et Jason-1. Ceci depuis la côte nord de l'Île de Marajó (pratiquement située sur l'Équateur) jusqu'aux environs de $02^{\circ}$ Nord. La position de l'axe de l'Amazone est choisie au milieu de son " panache » en se référant à la thèse de LE BARS (2010), et la valeur moyenne de l'altitude est de $+0,78 \mathrm{~m}$, ce qui demeure assez subjectif. Comme l'étude de la ligne d'eau du Bas Amazone ne porte, malheureusement, que sur l'année 2000, le raccordement de cette unique année avec la moyenne 1980/2009 s'est fait en fonction du carré de la distance entre l'océan et les diverses stations jusqu'à Santarém, procédé également un tantinet hasardeux, pour tenir compte de l'effet asymptotique du raccordement. Ceci conduit à dire que ces altitudes de la ligne d'eau (Tableau 2) peuvent différer quelque peu de leur vraie valeur. 


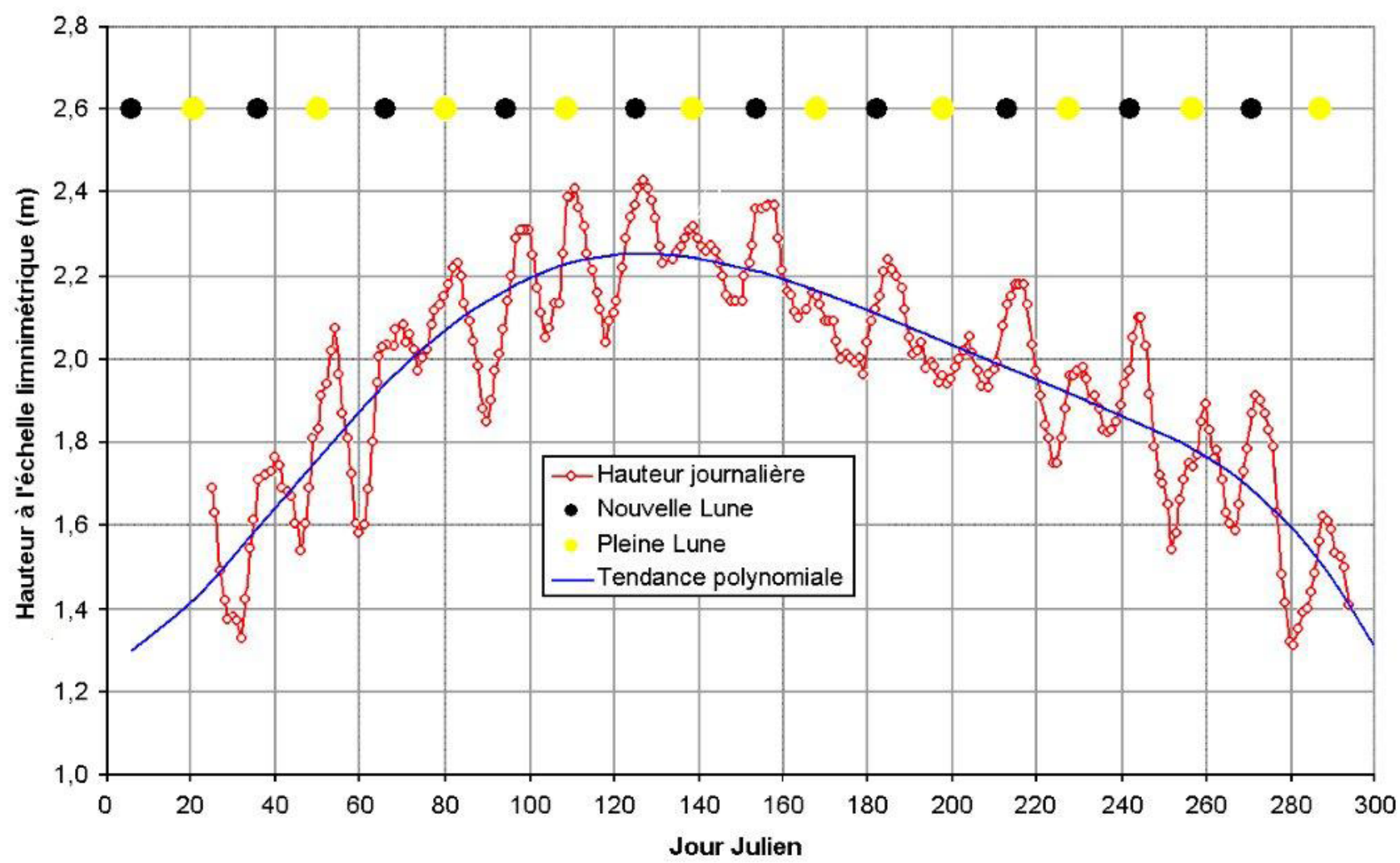

Figure 4. Répartition des hauteurs moyennes journalières de l'Amazone à Gurupá (année 2000). Distribution of daily mean heights of the Amazon at Gurupá (year 2000).

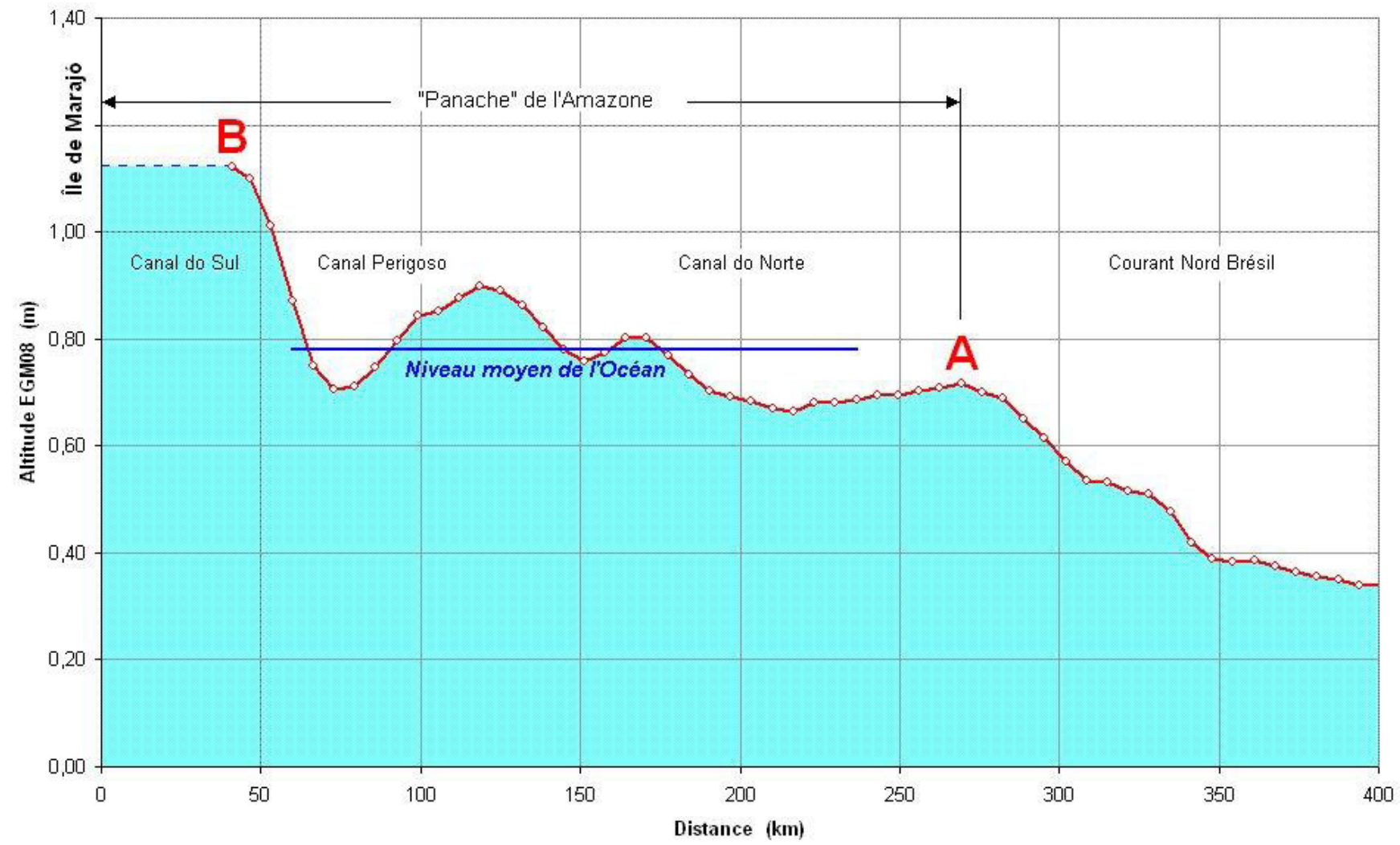

Figure 5. Profil de l'Océan Atlantique le long de l'orbite 202. Les points A et B correspondent aux points A et $B$ de la figure 3. Profile of the Atlantic Ocean along the orbit 202. Points A and B correspond to points $A$ and B in Figure 3. 
Tableau 2. Pente de la ligne d'eau du Bas Amazone.

Table 2. Slope of the Lower Amazon water surface.

\begin{tabular}{|c|c|c|c|c|c|c|}
\hline Station & $\begin{array}{c}\text { Distance } \\
(\mathrm{km})\end{array}$ & $\begin{array}{l}\text { Altitude du } \\
\text { Zéro de } \\
\text { l'Échelle } \\
\text { (m) }\end{array}$ & $\begin{array}{l}\text { Altitude de } \\
\text { l'Amazone } \\
\text { en } 2000 \\
\text { (m) }\end{array}$ & $\begin{array}{l}\text { Correction } \\
\text { (m) }\end{array}$ & $\begin{array}{c}\text { Altitude } \\
\text { moyenne de } \\
\text { l'Amazone } \\
\text { (m) }\end{array}$ & $\begin{array}{l}\text { Pente de } \\
\text { l'Amazone } \\
\left(\mathrm{mm} \bullet \mathrm{km}^{-1}\right)\end{array}$ \\
\hline Santarém & & 2,95 & 6,83 & $-0,21$ & 6,62 & \\
\hline & 176,5 & & & & & 10 \\
\hline Prainha & & 1,14 & 4,93 & $-0,13$ & 4,80 & \\
\hline & 110 & & & & & 10 \\
\hline Almeirim & & $-0,61$ & 3,75 & $-0,09$ & 3,66 & \\
\hline & 116,1 & & & & & 11 \\
\hline Gurupá & & 0,51 & 2,42 & $-0,06$ & 2,36 & \\
\hline & 180 & & & & & 3 \\
\hline Porto Santana & & $-2,89$ & 1,89 & $-0,02$ & 1,87 & \\
\hline & 281 & & & & & 4 \\
\hline $\begin{array}{l}\text { Océan } \\
\text { (orbite 202) }\end{array}$ & & & 0,78 & 0 & 0,78 & \\
\hline
\end{tabular}

Toutes les altitudes sont établies par rapport au géoïde déterminé par le modèle EGM08

\section{RÉSULTATS CONCERNANT LES PENTES DE LA LIGNE D'EAU DE L'AMAZONE ET DES RIOS NEGRO ET SOLIMÓES}

La figure 6, établie avec les tableaux 2, 3 et 4, représente le profil en long de l'Amazone (depuis l'océan), du Rio Negro et du Rio Solimóes (dans la partie brésilienne de leurs cours). Elle souligne la différence entre les lignes d'eau du Rio Negro et du Rio Solimóes avec la faible pente de la ligne d'eau du Rio Negro entre Barcelos et sa confluence.

\subsection{La ligne d'eau du Rio Negro}

Le tableau 3 récapitule les pentes des différents biefs du Rio Negro.

Alors que tous les biefs présentent des pentes moyennes interannuelles supérieures à $10 \mathrm{~mm} \bullet \mathrm{km}^{-1}$, seul celui de Moura-Manas présente une pente moyenne de seulement $6 \mathrm{~mm} \bullet \mathrm{km}^{-1}$. Ceci du fait que « ...rigidement orienté NO-SE le Rio Negro occupe au NO de Manacapuru un fossé d'effondrement en angle de faille... Dans la vaste nappe d'eau dont la transgression flandrienne a provoqué la formation dans la dépression tectonique, le Rio Negro a édifié un delta. Sa forme est particulière, très allongée, à cause de la configuration même de la nappe d'eau, elle-même commandée par la tectonique... "(TRICART, 1977). Ceci rappelle, en moins vaste, le delta intérieur du Niger.

Ce peu de pente avait déjà été souligné par RECLUS (1894) qui écrivait " ...Le contraste le plus net des deux eaux se présente au confluent du rio Negro et du rio Branco, « rivière blanche", presque laiteuse, qui descend des savanes limitrophes de la Guyane anglaise. Les deux courants se longent comme deux fleuves distincts dans le même lit d'aval; pendant la crue de novembre, alors que le rio Branco roule par exception plus d'eau que le rio Negro, on reconnait distinctement le flot jusqu'à plus de 50 kilomètres en aval, et même on peut en discerner quelques traces à une faible distance au nord de Manaos ». Les 120 premiers kilomètres de ce delta du Rio Negro, en amont de Manaus, constituent l'Arquipélago das Anavilhanas (Archipel des Anavilhanas) où le lit mineur, large d'une vingtaine de kilomètres, est parsemé d'îles où "...les deux chenaux principaux se localisent le long des deux versants. Ils exercent, surtout celui de la rive gauche, quelques sapements qui déclenchent des glissements, ce qui les alimente en alluvions... " (TRICART, 1977). Ceci est la caractéristique géomorphologique d'une région peu pentue.

Utilisant un Acoustic Doppler Current Profilers (Profileur acoustique à effet Doppler - ADCP), deux jaugeages étaient réalisés les 5 et 14 mars 1995 à Paricatuba $(25 \mathrm{~km}$ en amont de Manaus), c'est-à-dire en période où la pente du Rio Negro est quasi nulle à cause du Rio Solimóes. Les résultats indiquent que la vitesse moyenne de l'eau y était très faible $\left(10 \mathrm{~cm} \bullet \mathrm{s}^{-1}\right)$ et que les filets d'eau vont un peu dans tous les sens. 


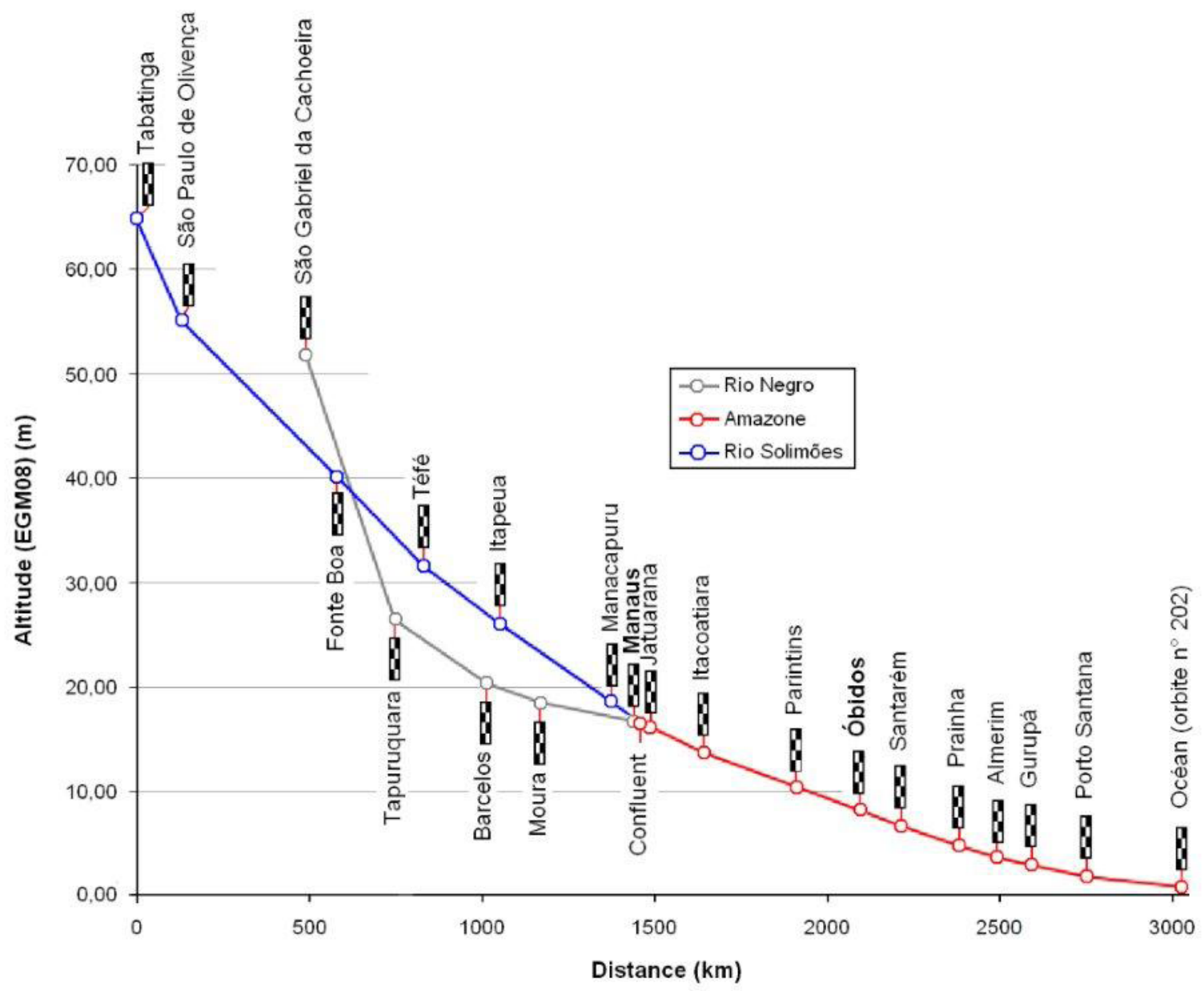

Figure 6. Profil en long de l'Amazone et de ses deux principaux tributaires, dans leur partie brésilienne. Longitudinal section of the Amazon and its two principal tributaries (within Brazilian territory).

Tableau 3. Pente de la ligne d'eau du Rio Negro.

Table 3. Slope of the Rio Negro water surface.

\begin{tabular}{|c|c|c|c|c|c|c|c|}
\hline \multirow[b]{2}{*}{ Station } & \multirow[b]{2}{*}{$\begin{array}{l}\text { Longueur } \\
\text { du bief } \\
\text { (km) }\end{array}$} & \multirow{2}{*}{$\begin{array}{l}\text { Altitude } \\
\text { moyenne } \\
\text { de l'eau } \\
\text { (m) }\end{array}$} & \multicolumn{5}{|c|}{ Pente de la ligne d'eau $\left(\mathrm{mm} \bullet \mathrm{km}^{-1}\right)$} \\
\hline & & & $\begin{array}{c}\text { Moyenne } \\
\text { inter } \\
\text { annuelle }\end{array}$ & $\begin{array}{c}\text { Moyenne } \\
\text { maximum } \\
\text { annuels }\end{array}$ & $\begin{array}{c}\text { Moyenne } \\
\text { minimum } \\
\text { annuels }\end{array}$ & $\begin{array}{c}\text { Maximum } \\
\text { absolu }\end{array}$ & $\begin{array}{c}\text { Minimum } \\
\text { absolu }\end{array}$ \\
\hline \multirow[t]{2}{*}{ São Gabriel } & & 51,64 & & & & & \\
\hline & 258 & & 83 & 87 & 79 & 89 & 75 \\
\hline Tapuruquara & 267 & & 36 & 42 & 31 & 46 & 28 \\
\hline \multirow[t]{2}{*}{ Barcelos } & & 20,54 & & & & & \\
\hline & 155 & & 14 & 33 & 3 & 42 & $-2(0,6)$ \\
\hline \multirow[t]{2}{*}{ Moura } & & 18,32 & & & & & \\
\hline & 273 & & 6 & 16 & 1 & 21 & $-1(0,4)$ \\
\hline
\end{tabular}

En rouge : dénivelée négative (l'altitude de l'aval est supérieure à l'altitude de l'amont

$-1(0.4)$ : -1 , pente négative maximale observée sur la série de 30 années

(0.4), pourcentage de jours de dénivelée négative sur le total de 10958 jours

(série de 30 années)

Toutes les altitudes sont établies par rapport au géoïde déterminé par le modèle EGM08 
Tableau 4. Pente de la ligne d'eau du Rio Solimóes et de l'Amazone.

Table 4. Slope of the Rio Solimóes and Amazon water surfaces.

\begin{tabular}{|c|c|c|c|c|c|c|c|}
\hline \multirow[b]{2}{*}{ Station } & \multirow[b]{2}{*}{$\begin{array}{c}\text { Longueur } \\
\text { du bief } \\
(\mathrm{km})\end{array}$} & \multirow{2}{*}{$\begin{array}{l}\text { Altitude } \\
\text { moyenne } \\
\text { de l'eau } \\
(\mathrm{m})\end{array}$} & \multicolumn{5}{|c|}{ Pente de la ligne d'eau $\left(\mathrm{mm} \cdot \mathrm{km}^{-1}\right)$} \\
\hline & & & $\begin{array}{c}\text { Moyenne } \\
\text { inter } \\
\text { annuelle }\end{array}$ & $\begin{array}{c}\text { Moyenne } \\
\text { maximum } \\
\text { annuels }\end{array}$ & $\begin{array}{c}\text { Moyenne } \\
\text { minimum } \\
\text { annuels }\end{array}$ & $\begin{array}{l}\text { Maximum } \\
\text { absolu }\end{array}$ & Minimum \\
\hline \multirow[t]{2}{*}{ Tabatinga } & & 64,74 & & & & & \\
\hline & 130 & & 74 & 81 & 63 & 100 & 50 \\
\hline \multirow[t]{2}{*}{ São P. de Olivença } & & 55,16 & & & & & \\
\hline & 451 & & 33 & 37 & 27 & 40 & 24 \\
\hline \multirow[t]{2}{*}{ Fonte Boa } & & 40,15 & & & & & \\
\hline & 249 & & 35 & 42 & 24 & 47 & 19 \\
\hline \multirow[t]{2}{*}{ Tefé } & & 31,66 & & & & & \\
\hline & 223 & & 24 & 28 & 18 & 31 & 15 \\
\hline \multirow[t]{2}{*}{ Itapeua } & & 26,06 & & & & & \\
\hline & 320 & & 23 & 28 & 18 & 31 & 11 \\
\hline \multirow[t]{2}{*}{ Manacapuru } & & 18,59 & & & & & \\
\hline & 86 & & 24 & 30 & 17 & 56 & 7 \\
\hline \multirow{2}{*}{ Confluent R. Negro } & & 16,54 & & & & & \\
\hline & 30 & & 14 & 27 & 1 & 35 & 0 \\
\hline \multirow[t]{2}{*}{ Jatuarana } & & 16,11 & & & & & \\
\hline & 154 & & 16 & 22 & 7 & 26 & 1 \\
\hline \multirow[t]{2}{*}{ Itacoatiara } & & 13,50 & & & & & \\
\hline & 268 & & 12 & 15 & 7 & 19 & 5 \\
\hline \multirow[t]{2}{*}{ Parintins } & & 10,40 & & & & & \\
\hline & 180 & & 12 & 15 & 6 & 16 & 3 \\
\hline \multirow[t]{2}{*}{ Óbidos } & & 8,25 & & & & & \\
\hline & 122 & & 13 & 17 & 8 & 19 & 2 \\
\hline Santarém & & 6,62 & & & & & \\
\hline
\end{tabular}

Toutes les altitudes sont établies par rapport au géoïde déterminé par le modèle EGM08

\subsection{La ligne d'eau du Rio Solimôes et de l'Amazone}

Le tableau 4 présente les résultats concernant le profil du Rio Solimôes et de l'Amazone.

Sur le Rio Solimóes, la pente de la ligne d'eau décroît régulièrement jusqu'à Manacapuru, puis elle augmente légèrement $\left(\mathrm{de} 1 \mathrm{~mm} \bullet \mathrm{km}^{-1}\right)$ jusqu'à la confluence avec le Rio Negro, puis décroît à nouveau.

Le bief Óbidos-Santarém, de par le rétrécissement de l'Amazone à Óbidos (garganta do Rio Amazonas) montre une pente un peu plus forte que le bief Parintins-Óbidos, ce qui est normal.

L'altitude de Tabatinga a fait l'objet de plusieurs déterminations barométriques car c'est l'endroit où l'Amazone pénètre au Brésil. RECLUS (1894) donne $82 \mathrm{~m}$ en se référant à la carte Mapa do Rio Amazonas (Carte de l'Amazone) de José da Costa AZEVEDO (futur baron de Ladário et futur Ministre de la Marine brésilienne) mais indique également $80 \mathrm{~m}$ d'après AGASSIZ, $77 \mathrm{~m}$ d'après ORTON, $200 \mathrm{~m}$ d'après SPIX et MARTIUS, $97 \mathrm{~m}$ d'après CASTELNAU. LE COINTE (1935) indique également $82 \mathrm{~m}$ et d'autres documents signalent 60 ,
73 et $85 \mathrm{~m}$. Rappelons que le niveau moyen du Rio Solimóes à Tabatinga est $64,74 \mathrm{~m}$.

\subsection{Comparaison avec les pentes des lignes d'eau d'autres fleuves}

Voici les pentes de la ligne d'eau de quelques fleuves près de leur embouchure, classées par pente croissantes :

- La Volga (KOLUPAILA et PARDÉ, 1933) : $40 \mathrm{~mm} \bullet \mathrm{km}^{-1}$ (de la mer Caspienne à $410 \mathrm{~km}$ en amont);

- Le Danube (PARDÉ, 1957) : $60 \mathrm{~mm} \bullet \mathrm{km}^{-1}$ (de la mer à Nagymaros, 1690 km);

- Le Nil (FOURTAU, 1919) : $75 \mathrm{~mm} \bullet \mathrm{km}^{-1}$ (de la mer Méditerranée à la $2^{\mathrm{e}}$ cataracte, $1550 \mathrm{~km}$ );

- Le Mississipi (BAULIG, 1949) : $109 \mathrm{~mm} \bullet \mathrm{km}^{-1}$ (de l'entrée de son delta jusqu'à $920 \mathrm{~km}$ en amont);

- le Rhin (BRUNNACKER et al., 1982) : $137 \mathrm{~mm} \bullet \mathrm{km}^{-1}$ (de Bonn à Nimègue, $270 \mathrm{~km}$ ).

La pente de la ligne d'eau de l'Amazone est bien plus faible :

- De Santarém à l'océan $(855 \mathrm{~km}): 7 \mathrm{~mm} \bullet \mathrm{km}^{-1}$; 
- De Tabatinga à l'océan $(3068 \mathrm{~km}): 21 \mathrm{~mm} \bullet \mathrm{km}^{-1}$;

- De Tabatinga à Santarém $(2213 \mathrm{~km}): 26 \mathrm{~mm} \bullet \mathrm{km}^{-1}$,

ce qui pose problème pour expliquer le si fort débit du fleuve (voir 5. CONCLUSION).

\section{LA CONFLUENCE DU RIO SOLIMÓES ET DU RIO NEGRO. DISCUSSION}

Située aux portes de Manaus, la confluence est malheureusement dépourvue de station hydrométrique, celle de Manaus étant située à $14,5 \mathrm{~km}$ plus en amont, sur le Rio Negro.

Voici ce qu'écrivait RECLUS (1894) sur cette confluence : "... Au confluent du Solimões et du rio Negro, celui-ci, à l'onde noire un peu pâlie par les eaux blanches du rio Branco, se mêle plus promptement au courant du violent Amazone qui s'empare du flot de l'affluent par de vastes remous...". MEDEIROS MOREIRA (2010) indique " estando de acordo com o forte efeito conhecido na regiáo de remanso hidráulico causado na foz do rio Negro e provocado pelas águas do rio Solimóes " (qui est cohérent avec le fort effet connu dans la région, causé par le remous hydraulique à l'embouchure du Rio Negro et provoqué par les eaux du Rio Solimóes).

La figure 7 indique l'emplacement d'un jaugeage effectué à l'ADCP le 18 mars 1995 et la figure 8 montre les directions des filets d'eau. Il est curieux de constater que, bien que les eaux du Rio Negro ne se soient pas mélangées avec celles du Rio Solimóes (un peu en contradiction avec E. RECLUS), l'ensemble des filets d'eau présente une bonne homogénéité alors que la section de mesure est à la sortie même de la confluence.

L'examen des registres d'inspection des hydrologues de la CPRM (http://visualizador.ana.gov.br:8080/ VisualizadorWebLogic/) concernant la station de Jatuarana, (située à $30 \mathrm{~km}$ en aval de la confluence) montre que cette station est située sur une rive sujette à l'érosion et que les éléments de l'échelle limnimétrique sont bien souvent à remplacer ou à recaler.

La rupture de pente liée à la confluence se comprend facilement quand on examine la superposition (Figure 9) des limnigrammes de Moura, Manaus, Jatuarana et Manacapuru : l'altitude maximale annuelle du Rio Solimóes à Manacapuru est de même ordre de grandeur que celle du Rio Negro à
Moura avec une distance Manacapuru-confluent cinq fois plus faible que pour Moura-confluent. De plus, le module du Rio Solimôes $\left(133000 \mathrm{~m}^{3} \bullet \mathrm{s}^{-1}\right)$ est nettement supérieur à celui du Rio Negro (29 $\left.000 \mathrm{~m}^{3} \bullet \mathrm{s}^{-1}\right)$. Conséquence : le Rio Solimões va faire barrage hydraulique au Rio Negro et perturber la confluence.

L'hydraulique de la confluence même des rios Solimóes et Negro mériterait une étude un peu plus approfondie.

Pentes négatives (niveau aval plus haut qu'à l'amont)

Les quelques cas (à peine $1 \%$ des observations, dans un seul bief) se produisent sur les biefs entre Barcelos et Jatuarana, le plus souvent à la montée de la crue. Cela signifierait que l'écoulement pourrait changer de sens, localement, pendant quelques dizaines de jours.

Sans parler des relevés de hauteur d'eau fantaisistes et non détectés, ils peuvent être provoqués :

- par un temps de propagation dans le bief, non nul et mal adapté;

- par le remplissage ou la vidange des plaines d'inondation (várzeas);

- par des apports extérieurs au bief (Rio Branco);

- par de grosses anomalies de niveau sur le Rio Solimóes,

ce qui conduira localement à un régime non permanent progressivement varié.

\section{CONCLUSION}

Les nouvelles techniques de nivellement satellitaires nous permettent de déterminer, enfin, les altitudes du zéro des stations hydrométriques brésiliennes de l'Amazone et de ses deux composants principaux. Ceci devrait s'avérer une aide efficace à la création de modèles mathématiques destinés à l'étude de l'écoulement sur ce bassin.

L'exploitation des données de nivellement GPS en mode "dynamique " permettra certainement d'affiner la connaissance des lignes d'eau entre deux stations hydrométriques. Le nivellement en mode "dynamique " consiste, avec l'antenne GPS installée sur le toit du bateau (et en ayant déterminé la hauteur antenne-ligne de flottaison), à mesurer continuellement la position XYZ du bateau qui se déplace sur le cours d'eau, réalisant ainsi le profil en long de la ligne d'eau. Il reste maintenant à compléter la partie brésilienne et de faire de même sur les portions du bassin amazonien extérieur au Brésil (Bolivie, Pérou, Equateur, etc.). 


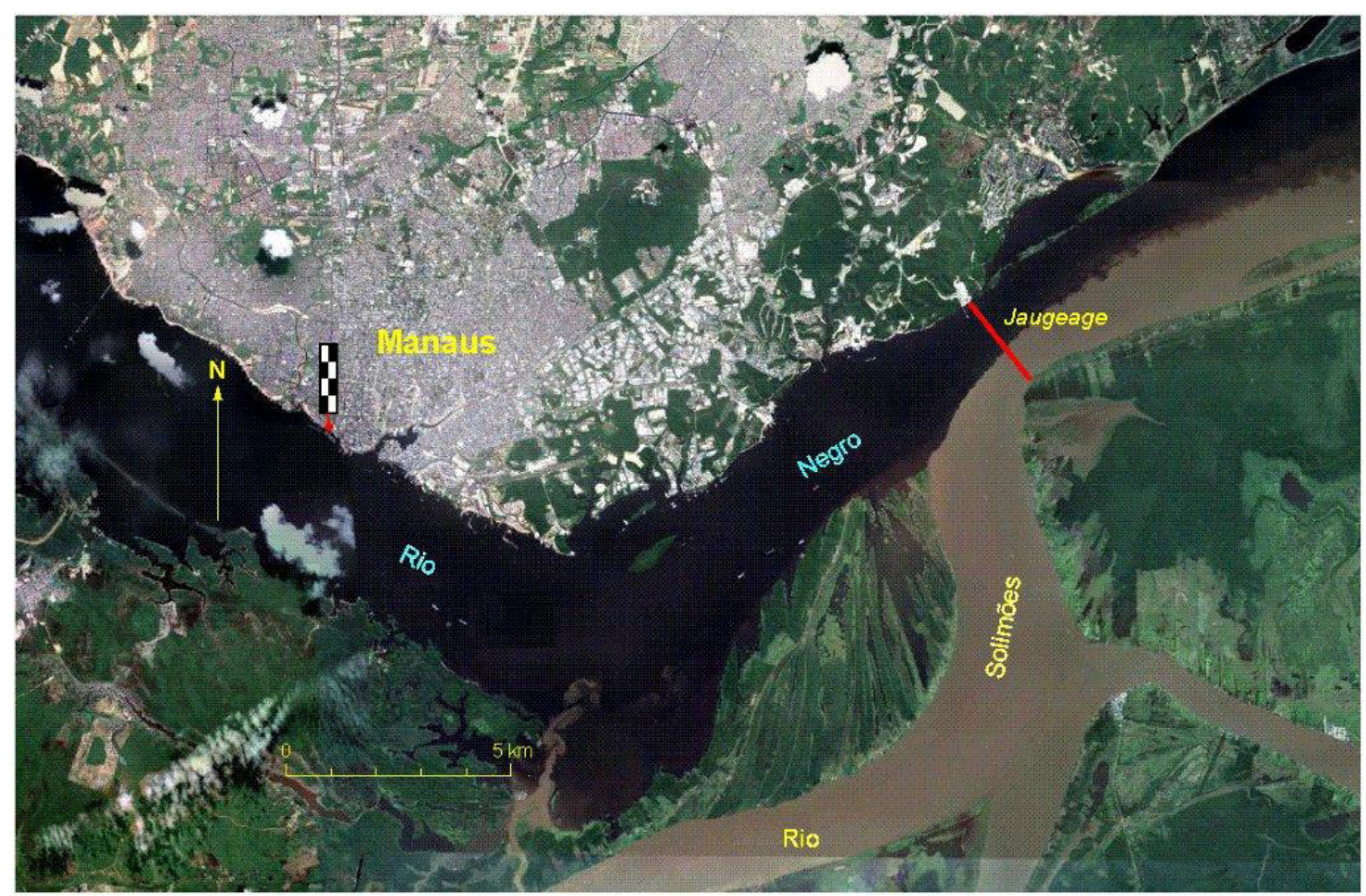

Figure 7. Localisation du jaugeage du 18 mars 1995 (débit : $86227 \mathrm{~m}^{3} \cdot \mathrm{s}^{-1}$ ) à la Confluence des Rios Negro et Solimóes. La ligne de partage des deux eaux est très nette, le mélange des eaux ne sera effectif que $180 \mathrm{~km}$ plus loin (image Google Earth, 2011).

Location of the gauging on 18 March 995 (flow rate: $86,227 \mathrm{~m}^{3} \cdot \mathrm{s}^{-1}$ ) at the confluence of the Rios Negro and Solimóes. The dividing line between the two waters is very clear; mixing water will be effective only $180 \mathrm{~km}$ away (Google Earth image, 2011).

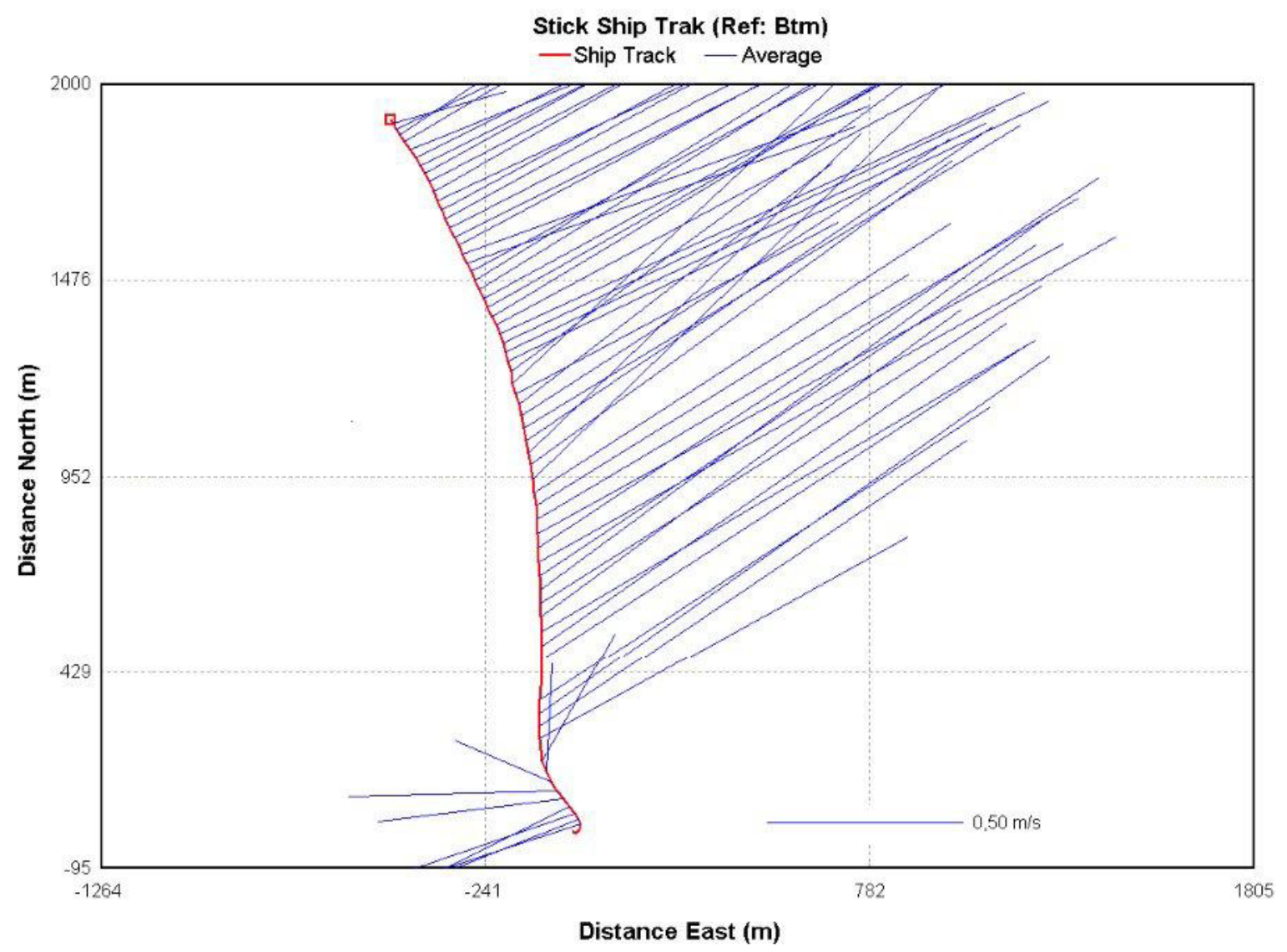

Figure 8. Jaugeage du 18 mars 1995 à la Confluence. Direction des filets d'eau. Gauging on 18 March 1995 at the confluence. Direction of water streams. 


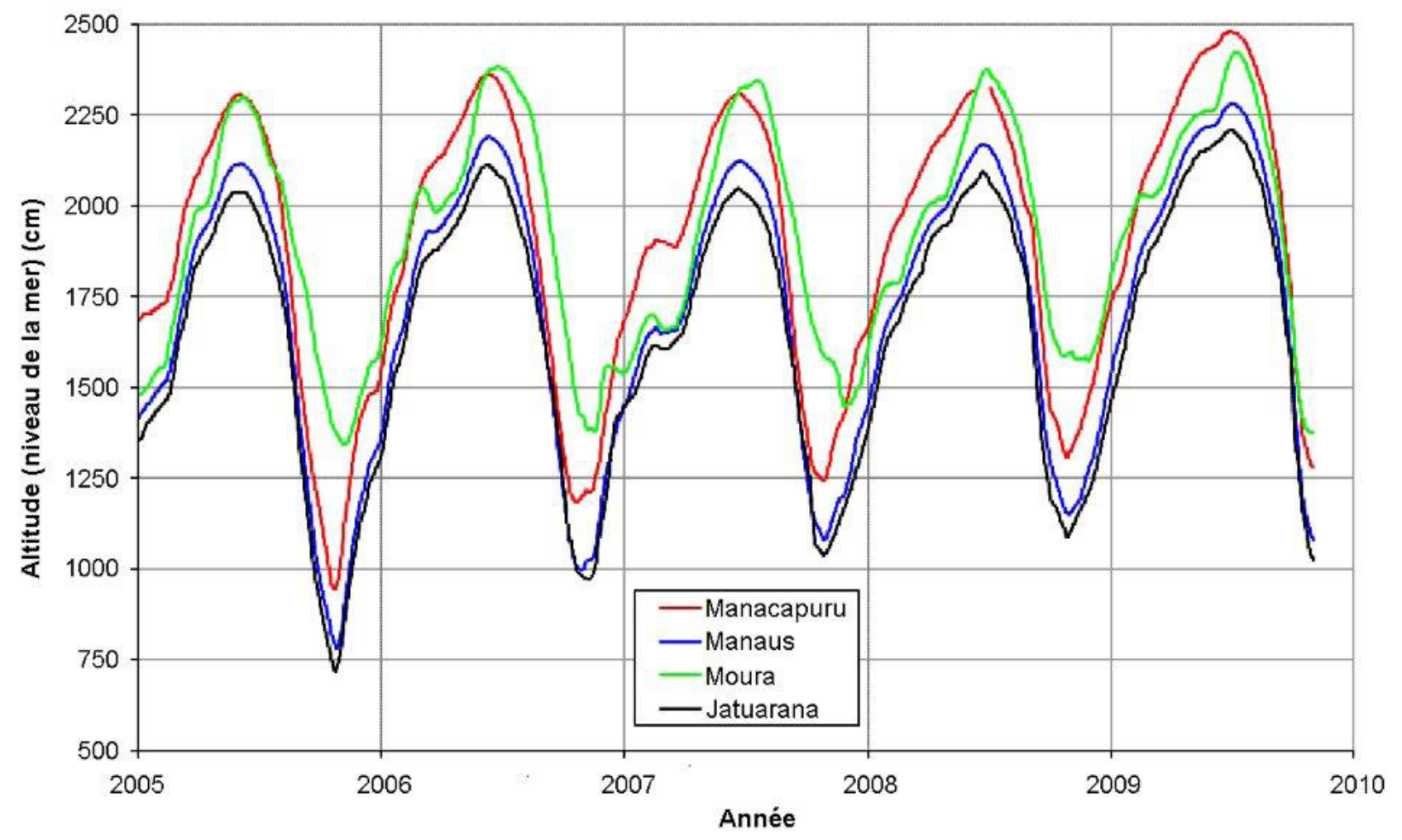

Figure 9. Superposition des limnigrammes de Moura, Manaus, Manacapuru et Jatuarana (période 2005-2009). Superposition of Moura, Manaus, Manacapuru and Jatuarana limnographs (for the period 2005-2009).

La très faible pente de la ligne d'eau de l'Amazone, en comparaison avec ses débits " monstrueux ", nous incite à adopter, pour ce fleuve, un schéma d'écoulement du genre décrit par LE COINTE (1935) «L'énorme volume d'eau provenant des pluies torrentielles qui s'abattent pendant plus de la moitié de l'année sur la partie supérieure du bassin amazonien et de la fonte estivale des neiges accumulées durant l'hiver sur les hauts sommets de la Cordillère des Andes ne trouve pas, dans le lit de très faible déclivité de l'unique collecteur, un déversoir de section suffisant et le flot qui arrive sans cesse se gonfle irrésistiblement et déborde, tout en se pressant vers l'océan " et par MOLINIER et al. (1996) : «Il ne s'agit pas seulement d'un écoulement classique de l'amont vers l'aval, mais aussi et surtout d'une poussée des eaux situées en aval par l'onde de crue des fleuves d'origine andine ».

Le fond du lit de l'Amazone est bien souvent en-dessous du niveau de l'océan : l'altitude du zéro de la station, combinée avec la profondeur tirée des jaugeages ADCP et de la hauteur d'eau à l'échelle le jour de ces jaugeages, nous confirment que le lit de l'Amazone est en-dessous de ce niveau zéro à un peu plus de $2000 \mathrm{~km}$ de l'estuaire. Voici les altitudes du fond du lit :

- À la section de mesure à l'embouchure (non représentée) située à cent cinquante kilomètres, environ, en amont de la trace au sol de l'orbite 202: - $42 \mathrm{~m}$;

- À Óbidos (point kilométrique (PK) 977) : - 53 m;

- À la confluence des Rios Solimões et Negro (PK 1619) : - $43 \mathrm{~m}$;

- À Manacapuru (PK 1705) : - $17 \mathrm{~m}$;

- À Itapeua (PK 2025) : - 43 m;

- À Téfé (PK 2248) : + $18 \mathrm{~m}$.

Ce qui, associé à l'existence à $200 \mathrm{~km}$ au large de la côte brésilienne d'un canyon sous-marin de l'Amazone (Figure 10) montrerait que, de Itapeua à l'Océan $(2025 \mathrm{~km}), 80 \%$ de l'écoulement de l'Amazone se fait en-dessous du niveau de l'océan. Cette poussée des eaux andines expliquerait peut-être que, malgré la pente extrêmement faible $\left(12 \mathrm{~mm} \cdot \mathrm{km}^{-1}\right)$ de ce tronçon, les vitesses de fond de l'eau soient bien souvent proches, voire supérieures, à un mètre par seconde et qu'en face 


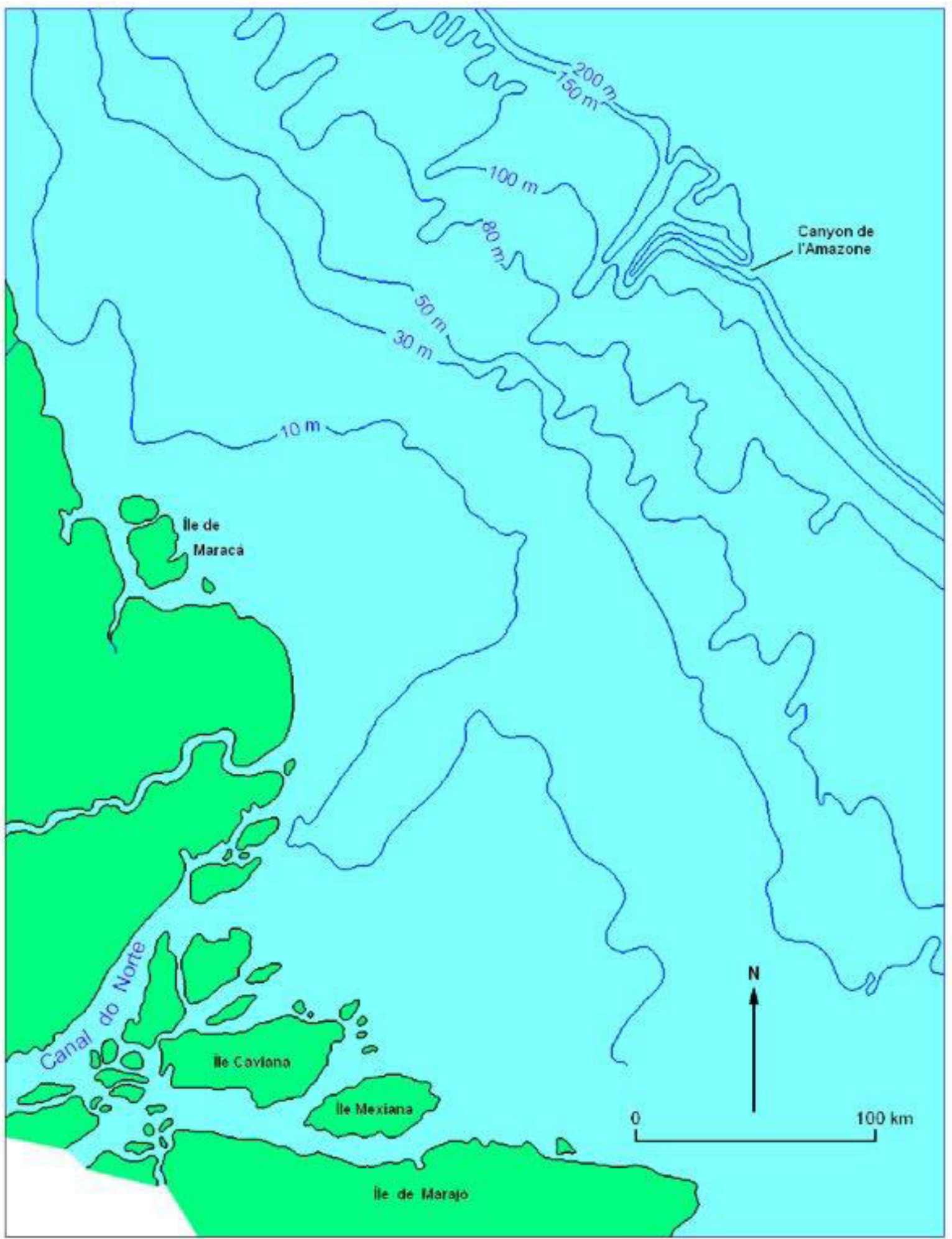

Figure 10. Localisation du canyon sous-marin de l'Amazone d'après la DHN (tiré de L. de Castro Soares, Géografia do Brasil, volume 3, regiáo Norte, Hidrografia, ed. IBGE).

Location of the Amazon River submarine canyon according to DHN (from L. de Castro Soares, Géografia do Brasil, volume 3, regiáo Norte, Hidrografia, ed. IBGE). 
de l'estuaire, la salinité normale de l'eau de mer (35\%) n'est atteinte également qu'à $200 \mathrm{~km}$ au large (COSTA DA SILVA et al., 2005; GORDEEV et al., 1992).

\section{REMERCIEMENTS}

Nous remercions F. FRAPPART du GET (Géosciences Environnement Toulouse), qui nous a fourni les coordonnées XYZ moyennes de la trace 202 des satellites TOPEX-Poseidon et Jason-1 pour la période 1993-2009, en face de l'embouchure de l'Amazone, ce qui nous a permis de raccorder le profil du fleuve à l'océan.

\section{RÉFÉRENCES BIBLIOGRAPHIQUES}

ARABELOS D.N. et C.C. TSCHERNING (2010). A comparison of recent Earth gravitational models with emphasis on their contribution in refining the gravity and geoid at continental or regional scale. J Geod., 84, 643-660.

BAULIG H. (1949). La vallée et le delta du Mississipi. Ann Geogr., 312, 325-334.

BERCHER N. (2008). Précision de l'altimétrie satellitaire radar sur les cours d'eau: développement d'une méthode standard de qualification de la qualité des produits alti-hydrologiques et applications. Thèse de doctorat d'AgroParisTech, Montpellier, France, 356 p.

BLITZKOW D. et A.C.O.C. DE MATOS (2009). EGM2008 and PGM2007. An evaluation for South America. Newton's Bull., 4, 79-89. Disponible à http://www.iges.polimi.it/ Newton/Newton_4/Report_A3_SAmerica.pdf.

BRUNNACKER K., M. LÖSCHER, W. TILLMANS et B. URBAN (1982). Correlation of the Quaternary terrace sequence in the Lower Rhine valley and Northern Alpine foothills of Central Europe. Quat. Res., 18,152-173.

CALlÈDE J., P. KOSUTH et E. de OLIVEIRA (2001). Établissement de la relation hauteur-débit de l'Amazone à Òbidos : méthode de la dénivelée normale à géométrie variable. Hydrol. Sci. J., 46, 451-463. http://iahs.info/ hsj/460/hysj_46_03_0451.pdf.

CALLÈDE J., Y. BOULVERT et J-P. THIÉBAUX (2010). Le bassin de l'Oubangui. IRD, Marseille (France). $1 \mathrm{CD}$, non paginé. ISBN 978-2-7099-1680-6.
CALMANT S. et F. SEYLER (2006). Continental surface water from satellite altimetry. C.R.Géosci., 338, 1113-1122.

CRÉTAUX J-F., S. CALMANT, V. ROMANOVSKY, F. PEROSANZ, S. TASHBAEVA, P. BONNEFOND, D. MOREIRA, C. K. SHUM, F. NINO, M. BERGÉNGUYEN, S. FLEURY, P. GEGOUT, R. ABARCA DEL RIO et P. MAISONGRANDE (2011). Absolute calibration of Jason Radar Altimeters from GPS kinematic campaigns over Lake Issykkul. Marine Geod., 34, 291-318.

COSTA DA SILVA A., M. ARAÚJO et B. BOULÈS (2005). Variação sazonal da estrutura de massas de água na plataforma continental do Amazonas e área oceânica adjacente (Variation saisonnière de la structure des masses d'eau de la plate-forme continentale de l'Amazone et de la zone océanique adjacente). Rev. Bras. Geof., 23, 145-157.

CROZIER R. (1938). Les profils en long des cours d'eau français. Ann Geogr., 47, 449-462.

DE CASTRO A.L.P. (2002). Nivelamento através do GPS. Avaliação e proposição de estratégias (Nivellement au GPS. Evaluation etproposition destratégies). Dissertação (mestrado) (Dissertation de Maîtrise), Universidade Estadual Paulista (Université d'État de São Paulo), Faculdade de Ciências e Tecnologia (Faculté des Sciences et Tecnologie), UNESP, Sáo Paulo, Brésil, 177 p. http://www4.fct.unesp.br/pos/ cartografia/docs/teses/d_castro_alp.pdf.

DE MARGERIE (1910). L'étude des profils en long des cours d'eau français. Ann Geogr., 19, 318-342.

DUQUENNE F. (2010). Évolution des références verticales. Dans : Journée Géodésie, IGN-Bureau des Longitudes, Paris, France, 14 octobre 2010, 69 p. http:// geodesie.ign.fr/contenu/fichiers/journeegeodesie/ presentations/7FDuquenne.pdf.

ERWES H. (1975). Experiences and accuracy of barometric levelling with THOMMEN altimeters. Dans : $1^{s t}$ Venezuelan Congress of Geodesy, Maracaibo, Venezuela, 1-6 décembre 1975, $28 \mathrm{p}$

FOURTAU R. (1919). Profil en long du Nil et de ses principaux affluents. Ann Geogr., 28, p. 400.

GORDEEV V.V., V.A. KONNOV et Y.V. KONNOVA (1992). Nitrogen forms in the Amazon River and estuary. Dans : Interactions of Biogeochemical Cycles in Aqueous Systems, Part 7, SCOPE/UNEP Sonderband, Hambourg, Allemagne, pp. 133-147. 
IBGE (Instituto Brasileiro de Geografia e Estatistica - Institut

Brésilien de Géographie et de Statistique) (2012). Sistema geodésico Brasileiro (Système géodésique brésilien)- Rede Altimétrica (Réseau altimétrique). Consulté le 20 décembre 2012, disponible à : http://www.ibge.gov.br/home/ geociencias/geodesia/altimetrica.shtm.

KOLUPAILA S. et PARDÉ M. (1933). Le régime des cours d'eau de l'Europe orientale. Rev. Geogr. Alp., 21, 651-748.

KOSUTH P., D. BLITZKOW et G. COCHONNEAU (2006). Establishment of an altimetric reference network over the Amazon basin using satellite radar altimetry (Topex-Poseidon). Dans : Venice 2006 Symposium "15 years of progress in radar altimetry", $8 \mathrm{p}$.

KOSUTH P. (2007) Hydrologie et télédétection : Altimétrie, géodésie et gravimétrie appliquées à l'hydrologie. Dans : Rapport quadriennal 2003-2006 du Comité National Français de Géodésie et de Géophysique, XXIV Assemblée Générale de l'Union Géodésique et Géophysique Internationale, 2-13 juillet 2007, Pérouse, Italie. Sur CD : 329-351.

KOSUTH P., J. CALLÈDE, A. LARAQUE, N. FILIZOLA, J-L. GUYOT, P. SEYLER J-M. FRITSCH et V. GUIMARÃES (2009). Sea-tide effects on flows in the lower reaches of the Amazon River. Hydrol. Process., 23, 3141-3150, doi:10.1002/hyp.7387.

KOUBA J. (2009). A Guide to using International GNSS Service (IGS) products. Natural Resources Canada, Ottawa, Canada, 34 p. Disponible à : http://acc.igs.org/ UsingIGSProductsVer21.pdf.

LE BARS Y. (2010). Modélisation de la dynamique océanique barotrope dans l'estuaire et le plateau amazoniens. Thèse de Doctorat en Océanographie physique, Université Paul Sabatier, Toulouse III, France, 196 p.

LE COINTE P. (1935). Les crues de l'Amazone et les récentes modifications de leur régime. Ann Geogr., 44, 614-619.

LEICK A. (2003). GPS satellite surveying. JOHN WILEY \& SONS (Éditeurs), Hoboken, États-Unis, 464 p.

LEON J.G. (2006). Utilisation conjointe de l'altimétrie spatiale et de la mesure in situ pour la régionalisation du débit dans le bassin versant du Rio Negro. Thèse de Doctorat en Hydrologie spatiale, Université Paul Sabatier, Toulouse III, France, 206 p.

MEDEIROS MOREIRA D. (2010). Rede de referência altimétrica para avaliação da altimetria por satélites e estudos hidrológicos na regiāo amazônica (Réseau de référence altimétrique pour la validation de l'altimétrie par satellites et les études hydrologique dans la région amazonienne. Diplôme de Master, Université Fédérale de Rio de Janeiro, Brésil, 175 p.

MOLINIER M., J-L. GUYOT, V. GUIMARÁES et E. de OLIVEIRA (1996). Les régimes hydrologiques de l'Amazone et de ses affluents. Dans : L'Hydrologie Tropicale : Géoscience et Outil pour le Développement (Actes de la conférence de Paris, mai 1995). IAHS Publ. № 238, 209-222. http://horizon.documentation.ird.fr/exl-doc/ pleins_textes/divers09-03/010009811.pdf.

MONICO J.F.G. (2000). Posicionamento pelo NAVSTAR-GPS (Positionnement par NAVSTAR-GPS). UNESP (Éditeur), São Paulo, Brésil, 287 p.

PARDÉ M. (1957). Les crues du Danube. D'après un numéro spécial de la revue hongroise d'hydraulique et des publications yougoslaves et bulgares. Rev. Geogr. Alp., 45, 387-399.

PAVLIS N.K., S.A. HOLMES, S.C. KENYON et J.K. FACTOR (2008). An earth gravitational model to degree 3160: EGM 2008. Dans : European Geosciences Union, General Assembly, 13-18 avril 2008, Vienne, Autriche, $32 \mathrm{p}$.

RECLUS E. (1894) Nouvelle géographie universelle. HACHETTE (Éditeur), Paris, France, 19, 824 p. Disponible sous http://gallica.bnf.fr/ark:/12148/ bpt6k31525j.

ROCHE M. (1963). Hydrologie de surface. GAUTHIERVILLARS (Éditeurs), Paris, France, 430 p.

SANTOS DA SILVA J., S. CALMANT, F. SEYLER, O. C. ROTUNNO FILHO, G. COCHONNEAU et W. J. MANSUR (2010). Water levels in the Amazon basin derived from the ERS 2 and ENVISAT radar altimetry missions. Rem. Sens. Environ., 114, 2160-2181.

RESSOURCES NATURELLES CANADA (2012). Modernisation du système de référence altimétrique. Sur http://www.rncan.gc.ca/sciences-terre/limite-geographique/ referencement-spatial/systeme-reference-elevation/ modernisation/5536\#cn-tphp.

SEYLER F., A. CAZENAVE, F. HUYNH, F. MERCIER, L. TOCQUEVILLE, S.CALMANT, M-P. BONNET, G. COCHONNEAU, M-C. GENNERO et M. MANGEAS (2007). Contribution of spatial altimetry to hydrology. The CASH Project. Dans : Rapport quadriennal 2003-2006 du Comité National Français de Géodésie et de Géophysique, 
XXIV Assemblée Générale de l'Union Géodésique et Géophysique Internationale, 02-13 juillet 2007, Pérouse, Italie. Sur CD : 281-309.

TRICART J. (1977). Types de lits fluviaux en Amazonie brésilienne. Ann. Geogr., 473, 1-54.

ZHANG M., H. LEE, C.K. SHUM, D. ALSDORF, F. SCHWARTZ, K-H. TSENG, Y. YI, C-Y KUO, H-Z. TSENG, A. BRAUN, S. CALMANT, N. FILIZOLA et F. SEYLER (2010). Application of retracked satellite altimetry for inland hydrologic studies. Int. J. Rem. Sens., 31, 3913-3929. 